\title{
The history of the baryon budget
}

\section{Cosmic logistics in a hierarchical universe ${ }^{\star}$}

\author{
Y. Rasera and R. Teyssier
}

DAPNIA, Service d'Astrophysique, Bâtiment 709, L'Orme des Merisiers, 91191 Gif-sur-Yvette Cedex, France

e-mail: romain.teyssier@cea.fr

Received 22 March 2005 / Accepted 3 June 2005

\section{ABSTRACT}

\begin{abstract}
Using a series of high-resolution $N$-body hydrodynamical numerical simulations, we investigate several scenarios for the evolution of the baryon budget in galactic halos. We derive individual halo star formation history (SFH), as well as the global star formation rate in the universe. We develop a simple analytical model that allows us to compute surprisingly accurate predictions, when compared to our simulations, but also to other simulations presented in Springel \& Hernquist (2003b, MNRAS, 339, 312). The model depends on two main parameters: the star formation time scale $t_{*}$ and the wind efficiency $\eta_{\mathrm{w}}$. We also compute, for halos of a given mass, the baryon fraction in each of the following phases: cold disc gas, hot halo gas, and stars. Here again, our analytical model predictions are in good agreement with simulation results, if one correctly takes finite resolution effect into account. We compare predictions of our analytical model to several observational constraints and conclude that a very narrow range of the model parameters is allowed. The important role played by galactic winds is outlined, as well as a possible "superwind" scenario in groups and clusters. The "anti-hierarchical" behavior of observed SFH is reproduced well by our best model with $t_{*}=3 \mathrm{Gyr}$ and $\eta_{\mathrm{w}}=1.5$. We obtain in this case a present-day cosmic baryon budget of $\Omega_{*} \simeq 0.004, \Omega_{\text {cold }} \simeq 0.0004, \Omega_{\text {hot }} \simeq 0.01$ and $\Omega_{\text {back }} \simeq 0.02$ (diffuse background).
\end{abstract}

Key words. galaxies: formation - galaxies: stellar content - cosmology: theory - methods: analytical - methods: numerical galaxies: evolution

\section{Introduction}

Thanks to recent advances in the observation of high-redshift star-forming galaxies and to the improved statistics in lowredshift galaxy surveys, it is now possible to have a quantitative view of the history of star formation in the universe, in a global, volume-averaged sense (Hughes et al. 1998; Steidel et al. 1999; Flores et al. 1999; Glazebrook et al. 1999; Yan et al. 1999; Massarotti et al. 2001; Giavalisco et al. 2004), but also for each galactic halo, in a statistical, mass-averaged sense (Heavens et al. 2004).

In the standard picture of galaxy formation, small-mass objects collapse first, when gravitational instability enters the non-linear regime. This so-called "hierarchical scenario" relies heavily on the observation of primordial density fluctuations imprinted on the Cosmic Microwave Background (Spergel et al. 2003). These first generation objects eventually form stars, the so-called population III stars, whose properties are likely to differ strongly from present-day galactic stars. The contribution of population III stars to star formation history in the universe is currently a matter of debate. They most likely contribute to a prompt initial enrichment of the intergalactic

* Appendices A, B and C are only available in electronic form at http://www.edpsciences.org medium, as well as to a possible early reionization epoch, but they may also cause strong negative feedback on the efficiency of star formation at this very early epoch. In this paper, we only address star formation occurring inside galactic discs, concentrating on quiescent modes of star formation. This means that we only consider that atomic processes drive the cooling and subsequent catastrophic collapse of halo gas that ultimately leads to the formation of a centrifugally supported disc. Molecular processes that are relevant for first star formation or for molecular clouds formation inside galactic discs are neglected in this study.

Our main concern is, therefore, to follow the formation of the first generation of gas rich, rotating discs, and the subsequent merging hierarchy that leads to larger and larger discs, up to present-day spiral galaxies, as well as galaxy groups and clusters. The standard approach in the current galaxy formation scenario is to consider that hot gas virializes first into extended dark matter halo potential well. Depending on its Virial temperature this hot gas cools rather rapidly, loses pressure support and collapses to a point where centrifugal equilibrium sets in. It is inside these cold, rotating discs that star formation takes place at the end of a complex cascade of turbulent fragmentation and molecular cloud formation. On a cosmological scale, models of star formation still rely on heuristic recipes, partially 
based on observational constraints and partially based on theoretical arguments.

This rather simple picture is altered by feedback processes due to these stars. First of all, massive stars collectively create a strong UV background that photo-ionizes the intergalactic medium, preventing small mass halos from building their virialized, hot gas component, and altering their cooling efficiency. This means that, after the universe is re-ionized, we have to introduce a mass threshold (or equivalently a Virial temperature threshold) below which star formation is suppressed. This threshold allows us to distinguish between a diffuse component, defined by small mass dark matter halos with a very low gas fraction, and a star-forming component, defined by high mass dark matter halos where disc and star formation are possible. The diffuse component is often called "the Lyman alpha forest", or else "the smooth baryon background", in the literature. In the present paper, we name the other component "the star-forming halos". Following standard definitions in galaxy formation studies, we further divide the baryons sitting in these "galactic halos" into 3 different phases: hot gas, cold discs, and stars. The purpose of this paper is to compute the evolution of these 4 phases as precisely as possible, for the universe as a whole, as well as for individual dark matter halos, thus studying the baryon logistics.

A lot of other different feedback processes are currently under close examination in the literature, like supernovae-driven winds, young protostellar jets, AGN-driven jets and associated buoyant bubbles, and so on. Such outflows are actually observed in high redshift galaxies (Martin 1999). They consist of high-velocity fountains of ionized gas expelled from a parent, star-forming disc. These winds are likely to be caused by collective outbreaks of supernovae bubbles (de Avillez \& Berry 2001). Other energy sources are, however, possible, such as a central massive black hole or collective jet ram pressure from star-forming clouds. Regardless of their physical origin, these outflows are fundamental in explaining the metal enrichment in the intergalactic medium, as well as the high-metallicity observed in rich galaxy clusters. The modeling of such winds will also be addressed in this paper in a simplified way, and their impact on the star formation history in the universe as a whole will be computed. We investigate the impact of winds on the star formation history of individual star-forming halos in more detail in a follow-up paper.

Several approaches have been used to investigate the evolution of the baryons in the hierarchical model of galaxy formation. Numerical simulations (Cen \& Ostriker 1992; Navarro \& White 1993; Mihos \& Hernquist 1994; Katz et al. 1996; Gnedin \& Ostriker 1997; Thacker \& Couchman 2000; Springel \& Hernquist 2003a) and semi-analytic models (White \& Frenk 1991; Somerville \& Primack 1999; Kauffmann et al. 1999; Cole et al. 2000; Hatton et al. 2003a) have been the most popular techniques. In a recent paper, an analytical approach is proposed for computing the star formation history of the universe (Hernquist \& Springel 2003). Here, we also present a simple self-consistent analytical model. This model, validated on simulations, allows us to quickly compute the evolution of the 4 baryons components, for the Universe as a whole, and also for an individual average halo.
The paper is organized as follows: in the next section, we present numerical simulations of star formation in a $\Lambda$ CDM universe, based on our Adaptive Mesh Refinement code named RAMSES. We then describe a simple analytical model that allows us to compute the star formation history inside individual halos and in the universe as a whole. In Sect. 4, we compare our model to simulation results, showing that, once calibrated to our simulations, the model works very well in predicting the evolution of the 4 different baryon phases. We finally compare our model to several observational constraints. Our analytical approach allows us to efficiently explore the parameter space, which in our case is limited to a 2-dimensional space: star formation time $t_{*}$ versus wind efficiency $\eta_{\mathrm{w}}$. We finally compare our predictions to current observational constraints, and find a rather narrow parameter range.

\section{Simulations}

Our simulations were performed using the Adaptive Mesh Refinement code called RAMSES and described in detail by Teyssier (2002). The $N$-body solver is very similar to the ART code (Kravtsov et al. 1997), and the hydrodynamical solver is based on a second-order Godunov-type method called the MUSCL-Hancock scheme (Toro 1997). We evolve the collisionless dark matter particle distribution by solving the VlasovPoisson equation and the baryonic component by solving the Euler equation with gravity source terms. More details about our hydrodynamical solver and our refinement strategy are given in Appendix A. We also solve for heating and cooling source terms in the energy equation, assuming primordial $\mathrm{H}$ and He plasma photo-ionized by the Haardt \& Madau (1996) UV background. In dense and cold regions of the flow, we turn a fraction of the gas into collisionless "star" particles. This numerical approach is widely used in current galaxy formation studies (Cen \& Ostriker 1992; Navarro \& White 1993; Mihos \& Hernquist 1994; Katz et al. 1996; Gnedin \& Ostriker 1997; Thacker \& Couchman 2000). We briefly recall here the properties of our own implementation. We then describe the cosmological parameters, box sizes, and mass resolution we use in our three main simulation series.

\subsection{Star formation recipe}

We now describe our method for implementing star formation in the RAMSES cosmological code. It is based on a heuristic approach adopted in many, if not all, cosmological studies. For a complete review of various implementations, see Kay et al. (2002). Basically, one considers that star formation proceeds at a given time scale, written here $t_{*}$, in region where one or several physical criteria are fulfilled. In this paper, as well as in many previous other papers in the literature, we adopt a simple scheme to turn gas mass into star particles, adding a source term in the continuity equation

$$
\begin{aligned}
& \left(\frac{D \rho}{D t}\right)_{*}=-\frac{\rho}{t_{*}} \text { if } \rho>\rho_{0}(1+z)^{\alpha_{0}} \\
& \left(\frac{D \rho}{D t}\right)_{*}=0 \quad \text { otherwise, }
\end{aligned}
$$


where the threshold density may depend on redshift through index $\alpha_{0}$. The star formation time scale $t_{*}$ is proportional to the local free-fall time

$t_{*}=t_{0}\left(\frac{\rho}{\rho_{0}}\right)^{-1 / 2}$.

In the literature, one can basically find two different approaches: a density threshold constant in physical units, corresponding here to $\alpha_{0}=0$, and a density threshold constant in comoving units, corresponding here to $\alpha_{0}=3$.

This simple model of star formation has been discussed and criticized extensively in the literature (Kay et al. 2002): we recall here briefly its possible physical and observational origins. Spiral galaxies in our nearby universe are seen to form stars at a rate given by the Kennicutt law (Kennicutt 1998), similar to Eq. (1), with volume densities $\rho$ and $\rho_{0}$ replaced by average disc surface densities.

$\Sigma_{0}=10 M_{\odot} / \mathrm{pc}^{2}$ and $t_{0}=2 \mathrm{Gyr}$.

The physical origin of such a behavior is not clearly identified yet. One good candidate is the sustained, interstellar, selfgravitating turbulent cascade, which controls the mass flux between large scale filaments in the disc and small-scale starforming molecular clouds, at a rate given by the local (on large scale, though) free-fall time (Elmegreen 2002). Such a precise description of star formation is completely irrelevant for our present cosmological study. Using a "sub-cell approach", similar in spirit to the one developed by the fluid mechanics community and often named the $k-\epsilon$ method, our current modeling of star formation tries to mimic in a statistical sense the complex behavior of the interstellar medium.

Along the same lines, Yepes et al. (1997) and, more recently, Springel \& Hernquist (2003a) have developed a multiphase model of the interstellar medium based on early work by McKee \& Ostriker (1977) on molecular clouds evaporation within supernovae remnants hot bubbles. This multiphase model offers an interesting alternative to the previous turbulent model. It also gives the possibility of self-consistently computing the star formation parameters, which are

$n_{0}=0.1 \mathrm{~cm}^{-3}$ and $\alpha_{0}=0$ and $t_{0}=2.1 \mathrm{Gyr}$.

Recall that in this case, star formation is allowed in regions whose gas density lies above a physical density threshold. In cosmological simulations, however, the comoving density is usually preferred to define collapsed, high-density clumps where star formation is likely to occur. In Springel \& Hernquist (2003b) for example, Eq. (1) is augmented with the requirement that the local overdensity should exceed 200. This guarantees that star formation cannot occur in smooth regions of the cosmological flow, but only within collapsed, virialized halos.

In the present paper, we would like to explore a different option than the one used in Springel \& Hernquist (2003b). We use the following star formation density threshold

$n_{0}=0.036 \mathrm{~cm}^{-3}$ and $\alpha_{0}=3$.

This corresponds to a baryon overdensity threshold of $1.6 \times$ $10^{5}$. This approach was also adopted in earlier works on star formation history in the universe (Cen \& Ostriker 1992; Kay et al. 2002; Nagamine et al. 2004), with threshold overdensities ranging from 5 to $10^{5}$ depending on the authors. In our case, this is motivated by our AMR refinement scheme; $n_{0}(1+z)^{3}$ corresponds exactly to the density threshold triggering the maximum level of refinement $\ell_{\max }=5$. When baryons cool and settle down at the center of their host dark matter halos, the density does increase dramatically. Our AMR code describes this collapse accurately, by recursively adding new cells at the center of the halo. At the point when the maximum level of refinement has been reached, the numerical description of the collapse is not valid anymore. We then turn on star formation, as our sub-cell modelling of this incomplete collapse, which would have ultimately lead to the formation of one or several star-forming molecular clouds.

As soon as star formation is active, we create collisionless star particles of constant mass $m_{*}$. As we explain in Appendix A.2, this constant mass is chosen to be equal to the initial mass resolution in the gas distribution, in order to prevent spurious refinement or de-refinement triggered by star formation. Using a constant mass for our star particles also has the advantage of controlling the maximum number of star particles created at the end of the simulation. In order to solve for the star formation source term (Eq. (1)) with constant star particles mass, we need to adopt a stochastic approach, similar to the one proposed in Katz (1992). In star-forming cells, we generate $N$ equal mass star particles, where $N$ is drawn from a Poisson process, with probability

$P(N)=\frac{\lambda^{N}}{N !} \exp (-\lambda)$

and with parameter (or mean value)

$\lambda=\left(\frac{\rho \Delta x^{3}}{m_{*}}\right) \frac{\Delta t}{t_{*}}$.

These star particles are created at each time step, at the very center of their parent cell. They are given a velocity equal to the local fluid velocity, plus a random component that we take to be equal to the local sound speed in the gas. The corresponding mass, momentum, and internal energy is, of course, conservatively removed from the parent cell's. In the simulation presented here, the only free parameter is the star formation time scale at comoving threshold density, $t_{0}$.

\subsection{Parameters}

We assume a single cosmological model throughout this paper, the so called "concordance model", with a cold dark matter component with $\Omega_{\mathrm{m}}=0.3$, a baryon component with $\Omega_{\mathrm{b}}=0.04$ and a dark energy component with $\Omega_{\Lambda}=0.7$. The Hubble constant was set to $h=0.7$. The initial power spectrum is assumed to be Harrisson-Zeldovich with $n=1$ and a normalization constrained by $\sigma_{8}=0.93$. The exact functional form we use for the transfer function is given in Sugiyama (1995).

We performed several AMR simulations, varying the box size, the number of particles and the star formation time scale $t_{0}$. The density threshold for star formation was set to $n_{0}=$ $0.036 \mathrm{~cm}^{-3}$ and was held constant in comoving units. In our 
Table 1. Runtime parameters for the "convergence study" simulation suite. $L$ is the box length, $n_{0}$ the density threshold at $z=0, t_{0}$ the star formation time scale for density $n_{0}$, and $\alpha_{0}$ gives the evolution of the star formation time scale with redshift. $N_{\text {cell }}$ is the number of cells at the coarse level, $\ell_{\max }$ the level of refinement, $z_{\text {end }}$ the final redshift, $m_{\mathrm{DM}}$ the dark matter particle mass, and $\Delta x$ is the spatial resolution.

\begin{tabular}{ccccc}
\hline \hline Name & L10N64S30 & L10N128S30 & L10N256S30 & L10N512S30 \\
\hline$L\left(h^{-1} \mathrm{Mpc}\right)$ & 10 & 10 & 10 & 10 \\
$n_{0}\left(\mathrm{~cm}^{-3}\right)$ & 0.036 & 0.036 & 0.036 & 0.036 \\
$t_{0}(\mathrm{Gyr})$ & 30 & 30 & 30 & 30 \\
$\alpha_{0}$ & 3 & 3 & 3 & 3 \\
$N_{\text {cell }}$ & $64^{3}$ & $128^{3}$ & $256^{3}$ & $512^{3}$ \\
$\ell_{\max }$ & 5 & 5 & 5 & 5 \\
$z_{\text {end }}$ & 3 & 3 & 3 & 3 \\
$m_{\mathrm{DM}}\left(h^{-1} M_{\odot}\right)$ & $2.8 \times 10^{8}$ & $3.4 \times 10^{7}$ & $4.3 \times 10^{6}$ & $5.4 \times 10^{5}$ \\
$\Delta x\left(h^{-1} \mathrm{kpc}\right)$ & 4.9 & 2.4 & 1.2 & 0.6 \\
\hline
\end{tabular}

Table 2. Runtime parameters for the "high efficiency" simulation suite. The meaning of the symbols is the same as Table 1 .

\begin{tabular}{cccc}
\hline \hline Name & L100N256S3 & L10N256S3 & L1N256S3 \\
\hline$L\left(h^{-1} \mathrm{Mpc}\right)$ & 100 & 10 & 1 \\
$n_{0}\left(\mathrm{~cm}^{-3}\right)$ & 0.036 & 0.036 & 0.036 \\
$t_{0}(\mathrm{Gyr})$ & 3 & 3 & 3 \\
$\alpha_{0}$ & 3 & 3 & 3 \\
$N_{\text {cell }}$ & $256^{3}$ & $256^{3}$ & $256^{3}$ \\
$\ell_{\max }$ & 5 & 5 & 5 \\
$z_{\text {end }}$ & 0 & 2.5 & 5.5 \\
$m_{\mathrm{DM}}\left(h^{-1} M_{\odot}\right)$ & $4.3 \times 10^{9}$ & $4.3 \times 10^{6}$ & $4.3 \times 10^{3}$ \\
$\Delta x\left(h^{-1} \mathrm{kpc}\right)$ & 12 & 1.2 & 0.12 \\
\hline
\end{tabular}

notation, this translates into $\alpha_{0}=3$. This star formation threshold corresponds to the overdensity threshold triggering our last level of refinement $\ell_{\max }=5$.

Our simulation suite can be organized in 3 different sets. The first set was designed to perform a convergence study. We used a box size of $10 h^{-1} \mathrm{Mpc}$ with identical physical and cosmological parameters. We vary only the initial number of particles and grid cells from $64^{3}$ to $512^{3}$. Runtime parameters for this 'convergence study' simulations are summarized in Table 1.

The second set of simulations was designed to explore a "high efficiency" SFR scenario with the maximum dynamical range we can afford (Table 2). For that purpose we used 3 different box size: 1,10 , and $100 h^{-1} \mathrm{Mpc}$. All these simulations were performed using $256^{3}$ particles and the same number of grid cells. This translates into a dark matter particle mass of $4.3 \times 10^{3}, 4.3 \times 10^{6}$, and $4.3 \times 10^{9} h^{-1} M_{\odot}$. The third set of simulations is similar to the second one but for a "low efficiency" SFR scenario (Table 3).

The 2 last simulation sets span a huge dynamical range in mass, but each simulation is valid for a limited range of redshifts. When the typical scale of non linearity reaches the box size, the simulation has to be stopped: the simulated volume is not statistically representative of the universe as a whole, and spurious effects due to periodic boundary conditions become visible. The stopping redshift was chosen to be 5, 2.5, and 0 for a box size of 1,10 , and $100 \mathrm{~h}^{-1} \mathrm{Mpc}$, respectively.
Table 3. Runtime parameters for the "low efficiency" simulation suite. The meaning of the symbols is the same as Table 1.

\begin{tabular}{cccc}
\hline \hline Name & L100N256S30 & L10N256S30 & L1N256S30 \\
\hline$L\left(h^{-1} \mathrm{Mpc}\right)$ & 100 & 10 & 1 \\
$n_{0}\left(\mathrm{~cm}^{-3}\right)$ & 0.036 & 0.036 & 0.036 \\
$t_{0}(\mathrm{Gyr})$ & 30 & 30 & 30 \\
$\alpha_{0}$ & 3 & 3 & 3 \\
$N_{\text {cell }}$ & $256^{3}$ & $256^{3}$ & $256^{3}$ \\
$\ell_{\max }$ & 5 & 5 & 5 \\
$z_{\text {end }}$ & 0 & 2.5 & 5.5 \\
$m_{\mathrm{DM}}\left(h^{-1} M_{\odot}\right)$ & $4.3 \times 10^{9}$ & $4.3 \times 10^{6}$ & $4.3 \times 10^{3}$ \\
$\Delta x\left(h^{-1} \mathrm{kpc}\right)$ & 12 & 1.2 & 0.12 \\
\hline
\end{tabular}

We have not implemented feedback processes in the RAMSES code, but it is currently under development. As explained in the introduction, galactic winds are a key ingredient in computing the star formation history in the universe. In this paper, we use SPH simulations results obtained by Springel $\&$ Hernquist (2003b) to estimate the influence of winds in the overall baryon budget.

Three sets of AMR simulations performed by us using the RAMSES code ("convergence study", "high efficiency" and "low efficiency") and one set of SPH simulations (Springel \& Hernquist 2003b) compose the data we analyze and discuss intensively in this paper.

\subsection{Results}

We now present general results obtained by the RAMSES code for a typical cooling and star formation run. We would like to outline that current high-resolution numerical simulations qualitatively reproduce the general picture of galaxy formation: fast cooling gas builds up centrifugally supported discs at the center of dark matter halos, in which star formation quietly proceeds. We will analyze our results more quantitatively in the next sections.

Figure 1 presents the simulated density field projected along one principal axis of the comoving periodic box. Run L10N512S30 is shown at redshift $z=2.8$. The projected gas density is shown with a logarithmically scaled color table. The 


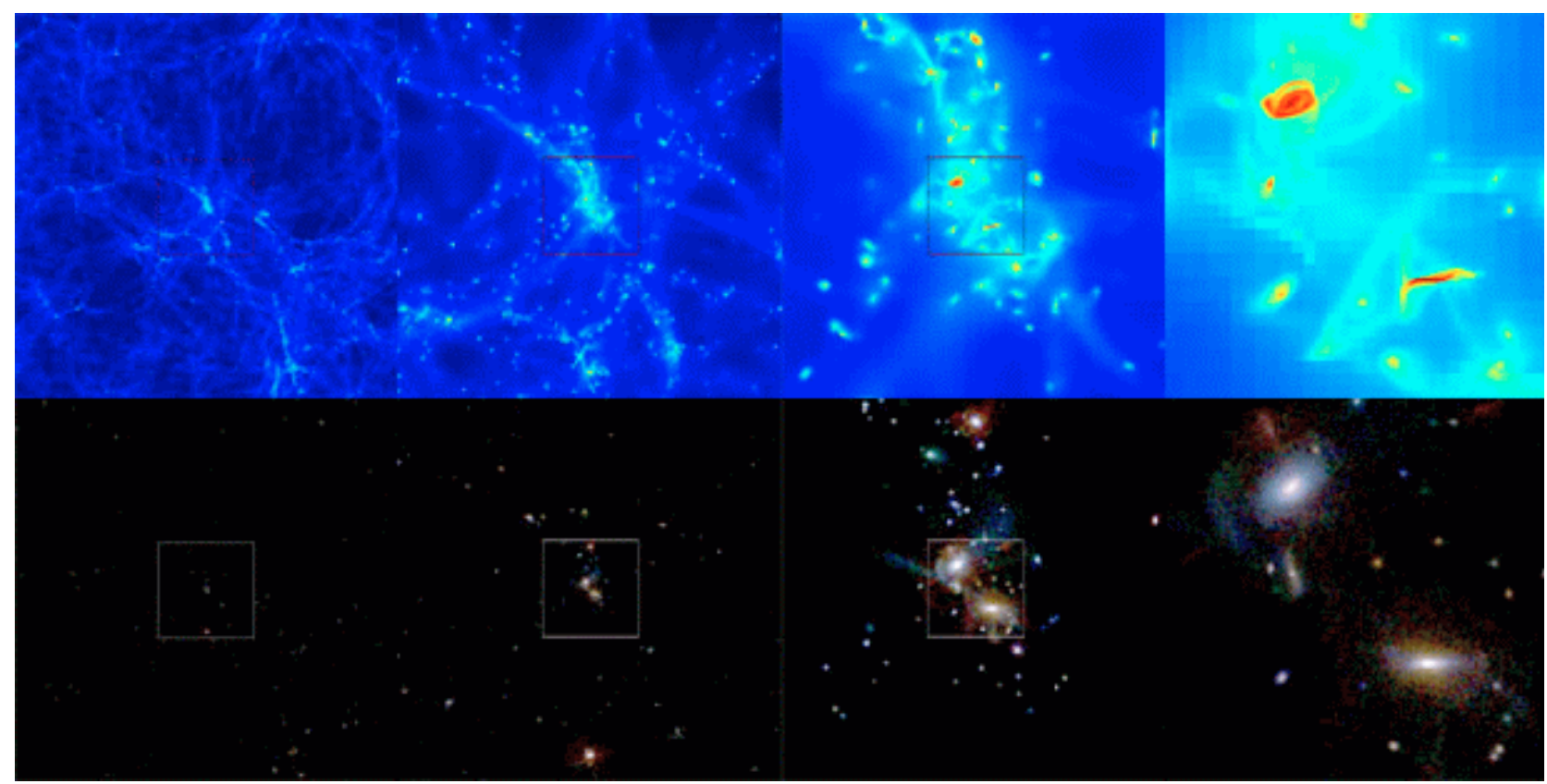

Fig. 1. Gas density (up) and stellar density (down) in our highest resolution run L10N512S30. The first map on the left of each row shows a projection of the full periodic volume. The squares in each map indicate the zoom region where the next image in the row is defined. For color map definitions, see text.

4 images show a zooming sequence, starting from the whole periodic box with the typical large scale filamentary structure, down to a particular region where 2 spiral discs are clearly visible.

The second set of images in Fig. 1 shows the same zooming sequence for the projected stellar density. These images were computed using a "true color" color scheme: stars are divided into 3 families according to the formation redshift of each star particle $\left(3<z_{\mathrm{f}}<4,4<z_{\mathrm{f}}<5\right.$ and $\left.5<z_{\mathrm{f}}\right) .3$ projected density maps are computed for each family. The 3 images are then combined using the RGB color scheme: "Red" stands here for old stars, "Blue" for young stars, and "Green" for intermediate formation redshift stars.

These rather spectacular images compare favorably with observed high redshift galaxies observed, for example, with the Hubble Space Telescope. We have to be careful in making definitive conclusions, because the spiral discs we observe in our simulations are highly underresolved. Figure 1 illustrates this point: the AMR grid structure clearly shows up in the highest resolution figure, demonstrating that these runs are not meant to resolve the internal structure of galactic discs. We are, however, confident about the computed baryon fraction inside each halo, as soon as the halo mass is greater than a few hundred particles.

The traditional way of describing the baryon density field is to divide it into 4 phases. This phase separation is apparent in Fig. 2, which shows the density-temperature histogram at $z=0$ for our large box run (100 $\left.h^{-1} \mathrm{Mpc}\right) \mathrm{L} 100 \mathrm{~N} 256 \mathrm{~S} 30$. The first regime occurs at low density $(\rho<200 \bar{\rho})$ and low temperature $\left(T<10^{5} \mathrm{~K}\right)$ in the phase space diagram. This is the diffuse intergalactic medium, also known as the Lyman alpha forest. The diffuse intergalactic UV flux is responsible for preventing this warm gas from collapsing into their parent dark matter halo.
The second regime occurs at high density $\left(\rho>10^{5} \bar{\rho}\right)$ and low temperature $\left(T<10^{5} \mathrm{~K}\right)$, and corresponds in our simulations to cold, centrifugally supported discs. This gas is termed here "cold gas", and is shown in Fig. 2 as the rightmost box. In this region, the quasi-neutral gas follows the very tight $\rho-T$ relation typical of high-density HI discs.

The remaining gas corresponds to shock-heated gas into virialized halos. We call it "hot gas" although, when cooling is efficient, its temperature never exceeds a few $10^{4} \mathrm{~K}$. This gas, in rough hydrostatic equilibrium, spans a large density range from $\rho \simeq \bar{\rho}$ in the outskirts of dark matter halos up to $\rho \simeq 10^{5} \bar{\rho}$ in the $\mathrm{X}$-ray emitting cores. This ionized gas is also rapidly cooling from the inside out, leading to the formation of the cold phase.

The last component in the baryon budget is the stellar phase. Stars originate from the highest density tail in the phase space diagram. In our model, star formation occurs above the overdensity threshold $\rho>10^{5} \bar{\rho}$, which corresponds to our cold phase.

Molecular cooling, as well as supernovae feedback, are not modeled here. The cold neutral gas is in reality decomposed into several components typical of the interstellar medium: molecular, very cold clouds embedded in hot, supernovae driven bubbles. The correct description of this multiphase interstellar medium is far beyond the possibilities of current cosmological simulations. The only possibility is to rely on subcell modeling, along the lines described in Yepes et al. (1997), for example, and Springel \& Hernquist (2003a). These authors notice that their multiphase modeling does not affect the overall baryon budget between the hot halo gas, the cold disc component, and the stellar fraction. The main effect of this multiphase approach is to modify the internal structure of the gaseous discs, altering the effective equation of state of the dense gaseous component. 


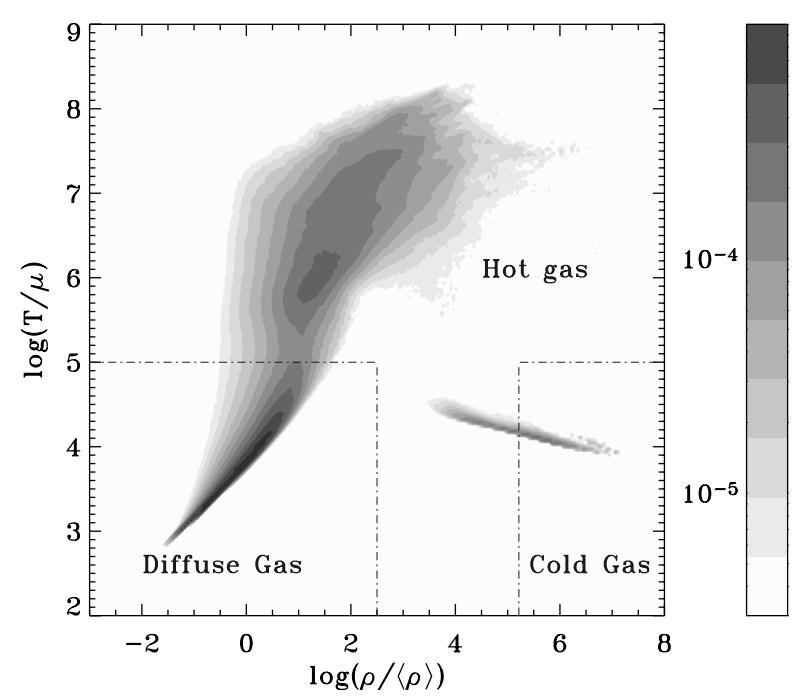

Fig. 2. The different baryon phases in the $\rho-T$ diagram. Gray contours show a mass-weighted histogram: the baryon mass fraction at a given density and temperature. Each region corresponds to a given phase (diffuse background, hot, or cold gas), as defined in the text.

The most important feature that is likely to affect the computed baryon budget is the mass resolution of the code. We have to carefully assess its effect on our results. Let us first examine Fig. 3, which shows a sequence of images of the projected gas density fields in a chosen region of the "convergence study" suite. Initial conditions were generated self-consistently for these 4 simulations, in order to recover the same large scale distribution. From the lowest resolution run with $64^{3}$ particles to start with, up to the highest resolution run with $512^{3}$ dark matter particles, one clearly sees the spectacular increase in small mass halos along the filaments and in the voids inbetween.

If we now examine the corresponding stellar density distributions in Fig. 3, we see that small halos appear devoid of stars. Small halos are indeed unsufficiently resolved to reach the high density contrast required to allow star formation. Cooling is also very likely to be affected by the poor resolution in these small halos. We will see in the next sections that for our simulations the minimum mass for a halo to host stellar particles is around 400 dark matter particles.

Another effect of mass resolution is also visible in the same figure. Large galactic discs obtained at a given resolution tend to fragment at higher resolution, leading to smaller discs with several orbiting satellites. These satellites are the remnants of progenitor halos, which form earlier at smaller mass. Insufficient resolution also affects the history of mass assembly inside galactic halos. Figure 3 shows that the color of a given galaxy is affected by the finite mass resolution. At low resolution, star formation is artificially delayed to late times and the galaxy appears blue, while at higher resolution, the correct star formation history is recovered and the same galaxy appears red. The purpose of this paper is to carefully estimate the effect of finite mass resolution on our predictions.

\section{Model}

In this section, we present a simple analytical model to compute the evolution of the baryon budget in the universe. Its purpose is to shed light on the complex behavior of our numerical simulations. Our analytical treatment of the cosmic star formation history is, of course, unaffected by finite resolution effect. It is, however, a crude model, so a careful comparison with numerical simulations is required to validate our approach.

This model differs from the "semi-analytic" modeling of galaxy formation, an approach pursued by several teams (White \& Frenk 1991; Somerville \& Primack 1999; Kauffmann et al. 1999; Cole et al. 2000; Hatton et al. 2003a). These models are based on a quite sophisticated treatment of the physics of galaxy formation; cooling, star formation, and spiral discs evolution are a few examples among the numerous ingredients in semi-analytical modeling.

In this paper, our goal is to compute the evolution in the mass fraction of the 4 different components in the baryon distribution: diffuse background, hot virialized plasma, cold neutral discs, and stars. We make predictions for the universe as a whole, but also for individual halos of a given mass. Semianalytical models, when coupled with $N$ body simulations, can predict the baryon history for an individual halo, based on the specific merging history at the origin of the halo hierarchical mass assembly. Our simple model allows us to compute only the average baryon history for halos of a given mass range.

In a recent paper, an analytical approach is proposed to compute the star formation history in the universe (Hernquist $\&$ Springel 2003). The proposed method was to use the PressSchechter theory for the dark matter halo statistics, together with the star formation rate as a function of halo mass, as measured in SPH numerical simulations. In the present paper, we develop a fully self-consistent analytical model, slightly more complex than the one proposed by Hernquist \& Springel (2003), but based on a similar approach. This self-consistency allows us to compute the mass fraction evolution of the 4 baryon components for the universe as a whole and also for an individual average halo.

\subsection{Halo model}

The method we use in this paper to compute the star formation history is based on what is usually called the halo model (Cooray \& Sheth 2002). The idea is to decompose dark matter and baryon density fields into a collection of virialized halos, whose distribution is described by the Press \& Schechter (1974) mass function. This approach was introduced to describe galaxy and dark matter clustering, using two additional ingredients: linear halo biasing theory and the Navarro et al. (1995) density profile (Ma \& Fry 2000; Seljak 2000). Later on, several authors used similar tools to compute the Sunyaev-Zeldovich power spectrum, as well as the mean Comptonization parameter (Cooray 2000; Refregier \& Teyssier 2002).

A feature common to most of these earlier works is the rather static picture they give of dark matter halo evolution. We propose to use a similar approach here, in order to compute 


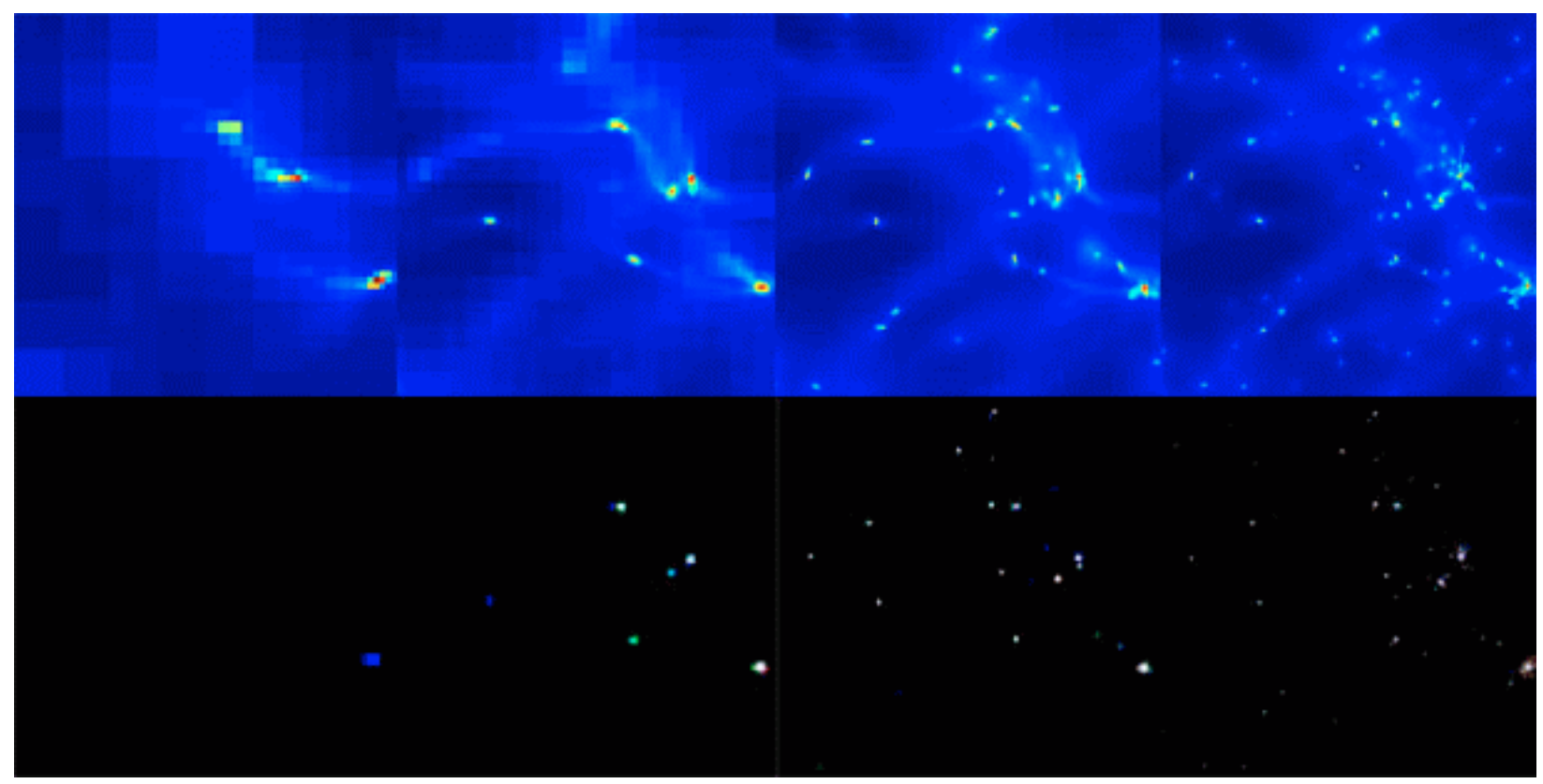

Fig. 3. Projected density maps (top) and projected stellar density (bottom) of our "convergence study" simulations series $\left(L=10 h^{-1} \mathrm{Mpc}\right)$. The image size is $1.25 h^{-1} \mathrm{Mpc}$ wide on a side. From left to right, the number of dark matter particles is increased from $64^{3}$, to $128^{3}, 256^{3}$, and $512^{3}$.

the history of star formation within each dark matter halo. Our methodology differs somewhat from earlier works, since it is based on a two-step approach.

In the first step, we compute the mass transfer rates between each phase using the static halo model. In the second step using these computed mass transfer rates, we solve for the mass fraction evolution equations, a system of first order Ordinary Differential Equations (ODE). Our analytical model is therefore based on the computation of the baryon logistics. In order to compute the baryon mass fraction locked up in stars, for example, we need to solve the complete set of ODE from a large redshift ( $z=200$ say) down to $z=0$.

In this paper, we define the halo mass $M$ as $M_{200}$, the total mass enclosed in radius $R_{200}$, where the mean overdensity (relative to the background density) is $\Delta=200$

$M=M_{200}=\frac{4 \pi}{3} \bar{\rho}(z) \Delta R_{200}^{3}$

This choice differs from earlier definitions, where $\Delta$ was either defined relative to the critical density or $\Delta$ was a function of redshift, as suggested by the spherical collapse model. As shown by Jenkins et al. (2001) and White (2002), these earlier definitions are not suited to the Press \& Schechter approach. Although they are based on physical principles, they destroy the self-similarity of the Press \& Schechter mass function.

In this paper, we therefore adopt $\Delta=200$ (relative to the mean background density) independent of redshift. We also use the halo circular velocity $V_{200}$ and the halo Virial temperature $T_{200}$ very often instead of the halo mass. The Virial temperature must not be considered as a true physical temperature, but rather as yet another mass parameterization. In this paper, the Virial temperature is defined as

$k_{\mathrm{B}} T_{200}=\frac{\mu m_{\mathrm{H}}}{2} \frac{G M_{200}}{R_{200}}$,

(with $\mu$ the mean molecular weight) and the circular velocity as

$V_{200}=\sqrt{\frac{G M_{200}}{R_{200}}}$.

In the 5 following sections, we are compute the cosmic rates between the 4 phases. If necessary the reader can go directly to Sect. 3.7.

\subsection{Minimal mass}

The first component in the baryon budget is the diffuse background. This may be the most important one, since it is the reservoir of fresh gas that will eventually feed star-forming halos at all epochs. It is usually called the Inter Galactic Medium (IGM) or the Lyman Alpha Forest. We need to give a precise definition of what we call 'diffuse background' in this paper.

The diffuse background is the baryon component associated to the dark matter halos with masses lower than the minimal mass $M_{\text {min }}$, below which cooling is inefficient and pressure forces prevent baryons to collapse in their potential wells. This minimal mass is therefore fundamental because it is the transition between "star-forming halos" and "diffuse" ones.

This Minimal Mass is taken to be the maximum between the Filtering Mass and the Minimal Cooling Mass. The filtering mass $M_{\mathrm{F}}$ is the minimal halo mass above which baryons are able to fall into their dark matter halo potential wells (see Appendix B). The minimal cooling mass $M_{\text {cool }}$ is the mass above which the gas is able to cool and therefore to form stars (see Appendix C). 


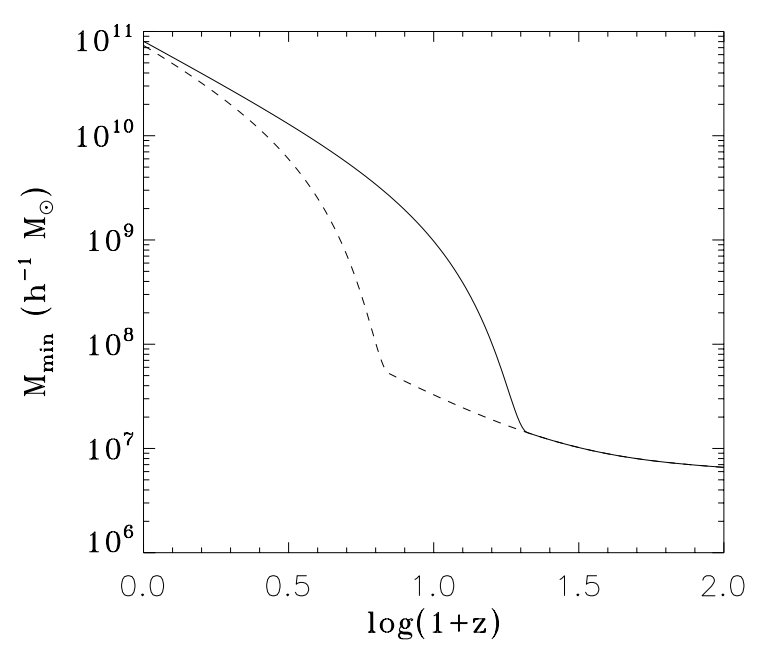

Fig. 4. Time evolution of the Minimal Mass $M_{\min }$ for two reionization scenarios: $z_{\mathrm{r}}=20$ (solid line) and $z_{\mathrm{r}}=6$ (dashed line). In both cases, the background temperature after reionization is set to $T_{\mathrm{r}}=6 \times 10^{3} \mathrm{~K}$. The Minimal Cooling temperature was also set to $T_{\text {cool }}=6 \times 10^{3} \mathrm{~K}$.

We implement this using a smooth function of both Virial temperatures

$T_{\min }=T_{\mathrm{F}}+T_{\text {cool }}$.

The Minimal Mass is finally computed using the Virial relation (Eq. (9)). In Fig. 4 we plot $M_{\min }$ as a function of redshift, for our two extreme reionization scenarios. At early times, this mass remains roughly constant, independent of redshift. As reionization proceeds, this Minimal Mass increases steadily, up to a rather large value $M_{\min } \simeq 10^{11} h^{-1} M_{\odot}$ today.

\subsection{Cosmic accretion rate}

Using the Press \& Schechter formalism, we now compute the mass fraction in the diffuse background and the mass fraction in star-forming halos. Since the Minimal Mass is considered here as the mass threshold between these two components, and assuming that the baryon fraction in each halo is equal to the universal one, we have

$f_{\text {hot }}=f\left(M>M_{\text {min }}\right)=f_{\mathrm{b}} \int_{v_{\min }}^{\infty} \sqrt{\frac{2}{\pi}} \exp \left(-v^{2} / 2\right) \mathrm{d} v$,

with

$v_{\min }=\frac{\delta_{\mathrm{c}}(t)}{\sigma\left(M_{\min }\right)} \quad$ and $\quad \delta_{\mathrm{c}}(t)=\frac{1.686}{D^{+}(t)}$,

where $D^{+}$is the linear growth factor and $\sigma\left(M_{\min }\right)$ the variance of the density field smoothed at the Minimal Mass scale. The rate at which baryons are transferred from the diffuse background to star-forming halos is computed by taking the time derivative of the previous equation

$\dot{f}_{\mathrm{acc}}=\frac{\mathrm{d} f_{\mathrm{hot}}}{\mathrm{d} t}=-\frac{\mathrm{d} f_{\mathrm{back}}}{\mathrm{d} t}=-f_{\mathrm{b}} \dot{v}_{\min } \sqrt{\frac{2}{\pi}} \exp \left(-v_{\text {min }}^{2} / 2\right)$.

We then define the Cosmic Accretion Rate of fresh diffuse gas into star-forming halos by

$\dot{f}_{\text {acc }}=\omega_{\text {acc }} f_{\text {back }}$ where the accretion rate, in units of $\mathrm{Gyr}^{-1}$, is given by

$\omega_{\text {acc }}=-\dot{v}_{\min } \sqrt{\frac{2}{\pi}} \frac{\exp \left(-v_{\min }^{2} / 2\right)}{\operatorname{erfc}\left(v_{\min } / \sqrt{2}\right)}$.

This last equation gives the mass accretion rate of diffuse gas into star-forming halos in the general case, for which the mass fraction in the diffuse component is allowed to vary from its canonical value. Note that this accretion rate has nothing in common with the traditional mass accretion rate on a given halo (Lacey \& Cole 1993). This rate gives the fraction of fresh diffuse gas dispatched among all star-forming halos. This fresh gas in assumed to be transferred exclusively to the hot plasma component. The two variables $f_{\text {back }}$ and $f_{\text {hot }}$ refer, therefore, to the total mass fraction in the background and to the total mass fraction in the hot component, both integrated over the PS distribution.

It is also possible to compute the Cosmic Accretion Rate on a halo-by-halo basis, using the Extended Press Schechter theory (Bond et al. 1991; Lacey \& Cole 1993). This theory allows the progenitors mass distribution as a function of time to be computed, for a given parent halo mass $M_{0}$ up to the "halo formation time" $t_{0}$. The individual Cosmic Accretion Rates are very similar to the one computed for the whole universe. We follow the same procedure computing first the mass fraction in star-forming halos, assuming that each progenitor hosts a baryon fraction equal to the universal one

$f_{\text {hot }}\left(M_{0}, t_{0}\right)=f_{\mathrm{b}} \int_{v_{\min }}^{\infty} \sqrt{\frac{2}{\pi}} \exp \left(-v^{2} / 2\right) \mathrm{d} v$,

with this time

$v_{\min }\left(M_{0}, t_{0}\right)=\frac{\delta_{\mathrm{c}}(t)-\delta_{\mathrm{c}}\left(t_{0}\right)}{\sqrt{\sigma\left(M_{\min }\right)^{2}-\sigma\left(M_{0}\right)^{2}}}$.

The accretion rate is then computed exactly as for the previous case, using Eqs. (15) and (16) with, however, a different value for $v_{\min }$ given by Eq. (18). We want to stress again that we do not consider accretion of satellite halos on the most massive progenitor, which is the traditional way of computing the accretion rate. Here, we consider accretion of diffuse gas on all star-forming progenitors of the final halo.

This fresh gas contributes to filling up dark matter halos with hot, virialized gas. Hot gas coming from satellite halos is automatically accounted for in our formalism. If we assume very short cooling rates and instantaneous star formation, this Cosmic Accretion Rate is nothing but the Star Formation History of the universe. In a realistic case, star formation and cooling introduce a delay in the curve.

This cosmic accretion rate depends on the thermal history of the background gas, on the density power spectrum through $\sigma(M)$, and on the cosmological model through $D^{+}(t)$. Figure 5 shows the accretion rates (in $\mathrm{Gyr}^{-1}$ ) as a function of redshift for a $\Lambda \mathrm{CDM}$ universe and for different halo masses. The halo formation redshift was fixed to $z_{0}=0$.

For small mass halos, accretion stops abruptly as the Minimal Mass reaches the parent halo mass. This means that star-forming progenitors are no longer present in the halo. For halos more massive than $M_{\min }$, accretion remains active up to 


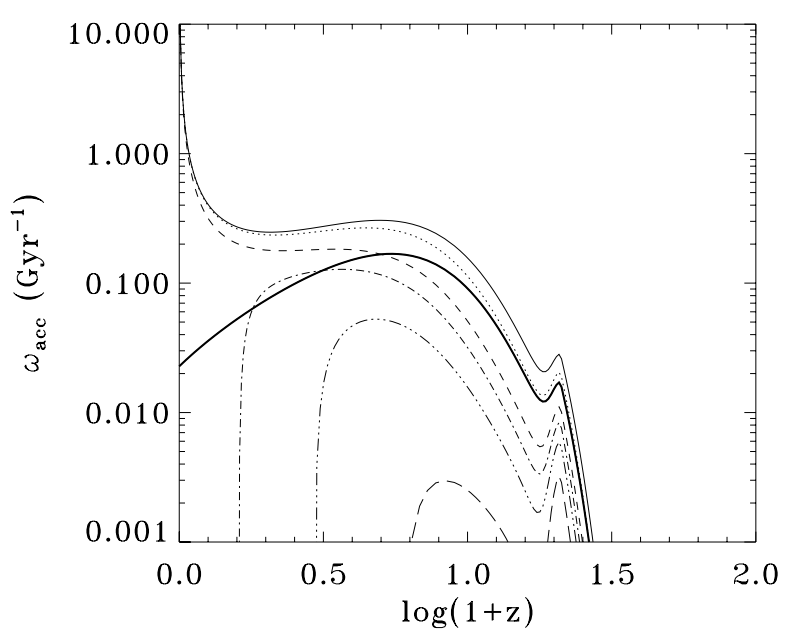

Fig. 5. Cosmic Accretion Rate for the $\Lambda \mathrm{CDM}$ cosmology with $z_{\mathrm{r}}=$ 20 for the universe as a whole (thick solid line) and for various halo masses (thin lines). Halo mass are, from top to bottom, $M_{0}=10^{13}$, $10^{12}, 10^{11}, 5 \times 10^{10}, 2.5 \times 10^{10}$ and $10^{10} h^{-1} M_{\odot}$. The halo formation redshift is set to $z_{0}=0$.

the halo formation redshift. The accretion rate actually diverges as $z \rightarrow z_{0}$, as all the remaining diffuse gas is accreted into the final virialized halo. This diffuse mass accretion rate remains, however, finite for $M_{0}>M_{\min }$, and we can compute its value as $z \rightarrow z_{0}$

$\dot{f}_{\mathrm{acc}}=-f_{\mathrm{b}} \sqrt{\frac{2}{\pi}} \frac{\dot{\delta}_{\mathrm{c}}\left(t_{0}\right)}{\sqrt{\sigma\left(M_{\min }\right)^{2}-\sigma\left(M_{0}\right)^{2}}}$.

\subsection{Cosmic cooling rate}

Our third baryon component (namely cold atomic gas in centrifugally supported discs) is progressively built up by accreting cooling gas into the very center of their parent dark matter halo. We need to estimate the global rate at which hot gas is transferred into this dense and cold component. Using EPS theory, we compute this rate for individual halo mass $\left(M_{0}, z_{0}\right)$. Note that we recover the results for the Universe as a whole by taking the limit $M_{0} \rightarrow+\infty$ and $z_{0} \rightarrow-1$.

We follow our basic methodology, assuming this time that all baryons are in the hot halo phase. Using our simple cooling model detailed in Appendix C, we compute the total amount of gas cooling from the hot halo component during a unit time interval by integrating the instantaneous cooling rate over the PS mass distribution from $M_{\min }$ to $M_{\max }$, the Maximal Cooling Mass. Indeed, above this mass (see Appendix C), the halo enters into the slow regime cooling. We therefore neglect this contribution. It gives

$\dot{f}_{\text {cool }}=f_{\mathrm{b}} \frac{1}{t_{\text {orb }}} \int_{v_{\text {min }}}^{v_{\max }} \sqrt{\frac{2}{\pi}} \exp \left(-v^{2} / 2\right) \mathrm{d} v$,

where $v_{\min }$ is defined by Eq. (18), $v_{\max }$ corresponds to the Maximum Cooling Mass $M_{\max }$

$v_{\max }\left(M_{0}, t_{0}\right)=\frac{\delta_{\mathrm{c}}(t)-\delta_{\mathrm{c}}\left(t_{0}\right)}{\sqrt{\sigma\left(M_{\max }\right)^{2}-\sigma\left(M_{0}\right)^{2}}}$, and $t_{\text {orb }}$ is the orbital decay time scale (see Appendix C). We then define the Cosmic Cooling Rate of hot halo gas into cold gaseous discs by

$\dot{f}_{\text {cool }}=\omega_{\text {cool }} f_{\text {hot }}$,

where the cooling rate, in units of $\mathrm{Gyr}^{-1}$, is given by

$\omega_{\text {cool }}=\frac{1}{t_{\text {orb }}} \frac{\operatorname{erfc}\left(\nu_{\min } / \sqrt{2}\right)-\operatorname{erfc}\left(v_{\max } / \sqrt{2}\right)}{\operatorname{erfc}\left(v_{\min } / \sqrt{2}\right)}$.

The Cosmic Cooling Rate depends on the cosmological model, on the thermal history of the background, and on the details of the cooling model. A similar model has been proposed by van den Bosch (2002), but in a different context.

\subsection{Star formation models}

In this simple analytical model, we completely discard the description of the gaseous discs. Predicting the disc sizes and surface density profiles obtained in the hierarchical scenario of structure formation is beyond the scope of this paper. We are only interested in the global baryon budget, and more precisely in the global star formation history.

We therefore consider star formation in a dark matter halo as a function of the total amount of cold gas in that halo. The star formation rate in each halo is simply given by $\dot{M}_{*}=$ $\omega_{*} M_{\text {cold }}$ with $\omega_{*}$ is the average star formation rate in that halo. In order to compute this average time scale from first principles, one needs to integrate the local, density dependent, star formation rate over the cold gas density PDF.

In this analytical model, however, we consider star formation models inspired by star formation recipes used in numerical simulations and by semi-analytical models (Somerville et al. 2001). The halo star formation rate is parameterized by

$\omega_{*}=\frac{1}{t_{*}}(1+z)^{\alpha_{*} / 2}$

where $t_{*}$ is the present day star formation time scale and $\alpha_{*}$ is the acceleration parameter. In the literature, two basic quiescent models are usually discussed in galaxy formation studies.

The first model, usually referred to as a "constant efficiency" model, assumes that the halo star formation time is a constant $\alpha_{*}=0$. This model corresponds to numerical simulations with a constant star formation density threshold.

The second model assumes that $\alpha_{*}=3$. It is usually called an "accelerated efficiency" model. The star formation time scale decreases with redshift (as the mean density of the Universe increases). This model corresponds to numerical simulations with a constant star formation overdensity threshold. It is also used in semi- analytical models to mimic starbursts triggered by mergers (Somerville et al. 2001).

We compute now the global star formation rate, using our basic methodology. Since the halo star formation rate, in our simple scenario, does not depend on halo mass, we can integrate over the EPS mass function, and obtain the Cosmic Star Formation Rate as

$\dot{f}_{*}=\omega_{*} f_{\text {cold }}$. 


\subsection{Cosmic winds}

The last ingredient in our model, but not the least, is the contribution of galactic winds to the overall baryon budget. It is a well known issue in current models of galaxy formation that without feedback processes, most baryons would end up in cold gas or stars, in contradiction with several observational constraints. This problem is known as the "overcooling problem" (Blanchard et al. 1992).

As discussed in the introduction, the exact nature of the dominant feedback process is still unknown. It is most likely that various processes are in competition, and their impact on baryons may vary as a function of halo mass. Following Springel \& Hernquist (2003b), we assume in our model that winds occur during star formation events, probably related to supernovae. We therefore assume that cold gas is ejected from the disc with a typical wind velocity $u_{\mathrm{w}}$ and with a typical outflow rate

$\dot{M}_{\text {wind }}=\eta_{\mathrm{w}} \dot{M}_{*}$.

The two additional parameters are $\eta_{\mathrm{w}} \simeq 1-5$, the wind efficiency, and $u_{\mathrm{w}} \simeq 200-500 \mathrm{~km} \mathrm{~s}^{-1}$, the wind velocity (Springel $\&$ Hernquist 2003b). These wind parameters are typical of observed outflows in star-forming galaxies (Martin 1999).

The fate of this ejected gas depends on the halo mass. If the wind velocity exceeds the escape velocity of the halo, the ejected gas leaves the halo into the diffuse background, from where, eventually, it will be accreted again. Such winds are referred to as "unbound". If, on the other hand, the halo is too massive, the ejected gas remains in the hot halo component, from where it will eventually cool again. Such winds are referred to as "bound".

Assuming again that all baryons are locked up into the cold component, we can compute the global wind outflow rate by integrating over the EPS mass function. We finally obtain the following equation, valid in the general case

$\dot{f}_{\text {wind }}=\omega_{\mathrm{w}} f_{\text {cold }}$.

where the outflow rate, in units of $\mathrm{Gyr}^{-1}$, is given by

$\omega_{\mathrm{w}}=\eta_{\mathrm{w}} \omega_{*}$.

We first compute the unbound fraction. It corresponds to winds emitted by halos whose escape velocity is smaller than the wind velocity. Using the EPS distribution, we get

$\zeta_{\mathrm{w}}=\frac{\operatorname{erfc}\left(v_{\min } / \sqrt{2}\right)-\operatorname{erfc}\left(v_{\mathrm{w}} / \sqrt{2}\right)}{\operatorname{erfc}\left(v_{\min } / \sqrt{2}\right)}$,

where $v_{\mathrm{w}}$ is defined by the "Wind Mass"

$v_{\mathrm{w}}\left(M_{0}, t_{0}\right)=\frac{\delta_{\mathrm{c}}(t)-\delta_{\mathrm{c}}\left(t_{0}\right)}{\sqrt{\sigma\left(M_{\mathrm{w}}\right)^{2}-\sigma\left(M_{0}\right)^{2}}}$.

This "Wind Mass" (the halo mass above which winds are bound) is related to the wind velocity by noticing that for typical dark matter halos, $v_{\text {esc }} \simeq 3 V_{200}$. Using the standard Virial relation (Eq. (9)), we obtain the wind Virial temperature

$k_{\mathrm{B}} T_{\mathrm{w}}=\frac{1}{18} \mu m_{\mathrm{H}} u_{\mathrm{w}}^{2}$.

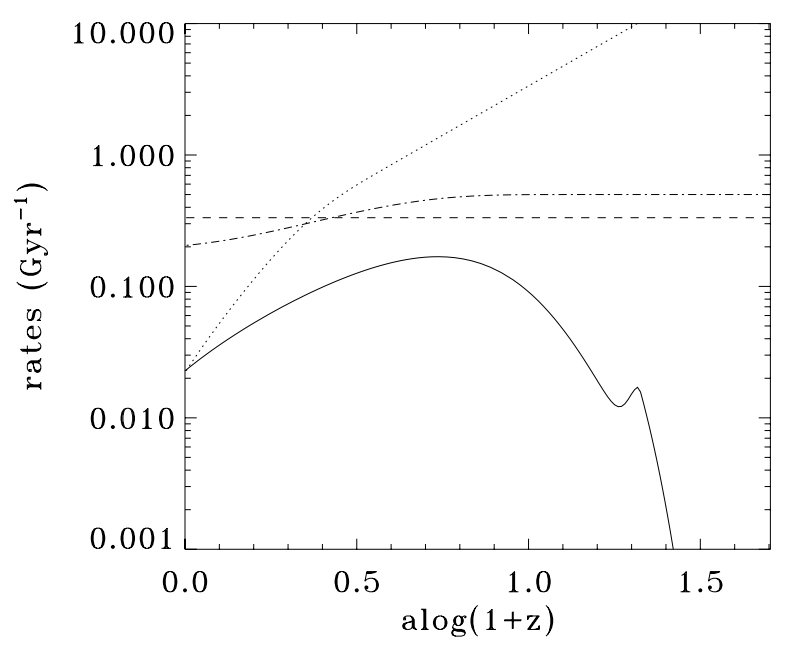

Fig. 6. Cosmic Accretion Rate (solid line), Cosmic Cooling Rate (dotted line), Cosmic Star Formation Rate (dashed line) and Cosmic Outflow Rate (unbound fraction, dot-dashed line) for the $\Lambda \mathrm{CDM}$ cosmology with the following model parameters: $z_{\mathrm{r}}=20, t_{*}=3 \mathrm{Gyr}$, $\alpha_{*}=0, T_{\mathrm{w}}=2 \times 10^{6} \mathrm{~K}$ and $\eta_{\mathrm{w}}=1.5$. These rates were computed using $M_{0}=+\infty$ and $z_{0}=-1$, and therefore corresponds to the universe as a whole.

The bound fraction is just $1-\zeta_{\mathrm{w}}$. The fate of the unbound gas depends now on the parent halo mass $M_{0}$. If $M_{0}>M_{\mathrm{w}}$, the gas is recycled into the halo diffuse component, and ultimately into the halo hot component as $z \rightarrow z_{0}$. If $M_{0}<M_{\mathrm{w}}$, the gas is lost into the intergalactic medium, outside the boundaries of the parent halo, and never comes back. Note that in the latter case, $\zeta_{\mathrm{w}}$ is always equal to one. This very crude model turns out to be surprisingly accurate in predicting results obtained by numerical simulations (see the following sections).

\subsection{The baryon supply chain}

We are now in a position to compute the baryon budget history. The last sections were devoted to computing mass transfer rates between our 4 baryon components. The mass fractions in each component are the independent variables in our problem: $f_{\text {back }}$, $f_{\text {hot }}, f_{\text {cold }}$ and $f_{*}$, referring respectively to diffuse background, hot gas, cold discs and stars.

The methodology we follow in this paper allows us to compute in advance 3 important mass transfer rates. These rates are $\omega_{\text {acc }}, \omega_{\text {cool }}$ and $\omega_{*}$, referring respectively to the Cosmic Accretion Rate, the Cosmic Cooling Rate and the Global Star Formation Rate. Our very crude wind model provides us with an additional parameter, namely the unbound fraction $\zeta_{\mathrm{w}}$. These various rates are plotted in Fig. 6 for our fiducial model and our notations are summarized in Table 4.

We have to solve a set of ordinary differential equations, with pre-computed transition rates between each component of our baryon supply chain

$\frac{\mathrm{d} f_{\mathrm{back}}}{\mathrm{d} t}=\zeta_{\mathrm{w}} \eta_{\mathrm{w}} \omega_{*} f_{\text {cold }}-\omega_{\mathrm{acc}} f_{\mathrm{back}}$,

$\frac{\mathrm{d} f_{\text {hot }}}{\mathrm{d} t}=\omega_{\mathrm{acc}} f_{\mathrm{back}}-\omega_{\mathrm{cool}} f_{\text {hot }}+\left(1-\zeta_{\mathrm{w}}\right) \eta_{\mathrm{w}} \omega_{*} f_{\text {cold }}$, 
Table 4. Main notations.

\begin{tabular}{cc}
\hline \hline$z_{\mathrm{r}}$ & reionization redshift \\
$t_{*}$ & star formation time scale \\
$\eta_{\mathrm{w}}$ & wind efficiency \\
$\zeta_{\mathrm{w}}$ & unbound wind fraction \\
$f_{*}$ & stellar fraction \\
$f_{\text {cold }}$ & cold gas fraction \\
$f_{\text {hot }}$ & hot gas fraction \\
$f_{\text {back }}$ & background gas fraction \\
$\omega_{*}$ & star formation rate \\
$\omega_{\text {cool }}$ & cooling rate \\
$\omega_{\text {acc }}$ & accretion rate \\
\hline
\end{tabular}

$\frac{\mathrm{d} f_{\text {cold }}}{\mathrm{d} t}=\omega_{\text {cool }} f_{\text {hot }}-\omega_{*} f_{\text {cold }}-\eta_{\mathrm{w}} \omega_{*} f_{\text {cold }}$,

$\frac{\mathrm{d} f_{*}}{\mathrm{~d} t}=\omega_{*} f_{\text {cold }}$.

If $M_{0}>M_{\mathrm{w}}$, one sees that the total baryon mass is conserved. Note that Eq. (32) should be modified if $M_{0}<M_{\mathrm{w}}$ : the wind contribution is set to 0 and therefore the total baryon mass in the parent halo is not conserved anymore. This is as expected, since winds are now escaping outside the parent halo boundaries.

Using any time integration method of sufficient accuracy, one can finally solve for the previous set of differential equations. We used in this work a Backward Euler scheme. Interestingly, one can solve formally the latter system using matrix exponentials. These types of equations are typical of galaxy-formation studies, like the early work of Tinsley (1980). More recently, Pei et al. (1999) have designed a similar approach, based on the observed galaxy luminosity functions, while here, our equations are based on EPS theory. Figure 7 shows the baryon budget evolution for our fiducial model. Before applying this analytical model to cosmological observations, we need to determine its validity range using high resolution numerical simulations.

\section{Simulations versus model}

We now compare our analytical predictions to the baryon budget history obtained in our high-resolution hydrodynamical simulations. Recall that we can compute analytically the baryon history for the universe as a whole, but also on a halo by halo basis. In order to make a careful comparison, we need to extract halos from the simulated density field. We use for that purpose a halo detection code based on the Spherical Overdensity algorithm (Lacey \& Cole 1993). We also need to carefully define the effective mass resolution of our simulations, with respect to star formation. This additional mass scale is a pure numerical artifact, that can be accounted for explicitly in our analytical model. Using this modified model, we will estimate how our results converge (or not) to the correct halo model predictions.

We need also to estimate the halo star formation time, (as defined in the previous section) in our numerical simulations. The star formation algorithm is based on a simple Schmidt law,

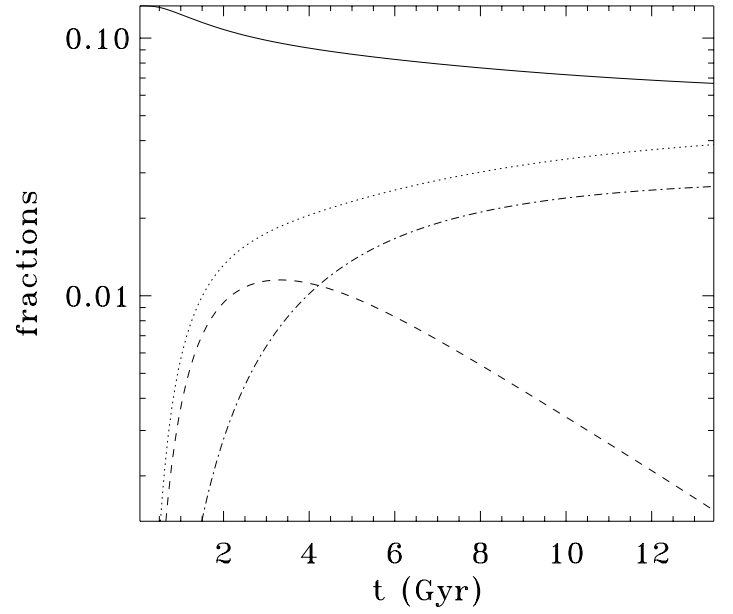

Fig. 7. History of the mass fraction in the diffuse background (solid line), in the hot halo gas (dotted line), in the cold discs (dashed line) and in stars (dot-dashed line) for the $\Lambda \mathrm{CDM}$ cosmology with the following model parameters: $z_{\mathrm{r}}=20, t_{*}=3 \mathrm{Gyr}, \alpha_{*}=0, T_{\mathrm{w}}=2 \times 10^{6} \mathrm{~K}$ and $\eta_{\mathrm{w}}=1.5$. These fractions were computed using $M_{0}=+\infty$ and $z_{0}=-1$, and therefore correspond to the universe as a whole.

with a specified (over-) density threshold. It is however more complex than the approach used in the halo model. We will show that both approaches can be related to each other, with a "shape factor" reflecting the probability distribution function of the cold phase density.

We finally need to estimate the unique free parameter in our analytical model: the orbital decay time scale. This sort of "model calibration" will be performed directly using our simulation results. We will also extend this "model versus simulation" comparison to other numerical data kindly provided to us by Springel \& Hernquist (2003b), for which galactic winds were included.

\subsection{Halo detection}

It is an absolute necessity to define a halo in a numerical simulation as it is defined in the theoretical model. As explained before, in order for the Press \& Schechter approach to be valid, the halo mass is the mass enclosed in radius $R_{200}$, enclosing an overdensity 200 times larger than the average background density. As noted by several authors (Jenkins et al. 2001; White 2002), this rather large region encloses dark matter particles which are not completely relaxed yet, and also several large satellites flying by. As a consequence, the halo mass turns out to be highly dependent on the exact algorithm used to detect automatically dark matter halos in the simulation.

These same authors suggest the following strategy: in a first step, halos are detected with a high density contrast $(\Delta=600$ is our choice here) by any classical algorithm (we use Spherical Overdensity in this paper). Since the density contrast is high, the detected region is in a well relaxed state and all algorithms agree more or less on the mass and on the number of detected halos. The halo center is defined as the center of mass of this high density region only. In a second step, the halo radius is 
increased up to $R_{200}$, in order to obtain the large halo mass required by the Press \& Schechter prediction.

We then compute for each individual halo the total stellar mass within $R_{200}$. Since we have stored the formation epoch of each individual star particle, we can also compute the complete star formation history of the parent halo. This last point is very important: we do not compute star formation rates and gas content of individual galaxies. Since each halo can host several galaxies (one central and several satellites), the galaxy baryon budget will be somewhat different from the halo baryon budget. Within the halo radius, we also compute the fraction of cold gas, defined as $T<10^{5} \mathrm{~K}$, and $\rho>10^{5} \bar{\rho}$. The remaining gas is considered as "hot gas", even though its temperature can be lower than the Virial temperature of the halo.

The effective star formation rate of each halo is computed by simply dividing the total amount of star created during the last $10 \%$ of the halo age by the elapsed time. The results presented in this section will be based on this analysis. Each individual halo baryon budget are averaged into mass bins, in order to compare with the halo model predictions.

\subsection{Mass resolution}

Results of cosmological simulations depend strongly on the mass resolution of the code. The two main numerical limitations are the box length and the number of particles. As shown in Fig. 3, the more particles we start with, the more small mass halos and galaxy satellites we obtain in the simulations. Since we are interested here in the global baryon budget, each individual halo must be able to allow gas to cool down and condense, and ultimately form stars. This is a more stringent requirement than just reaching the Virial overdensity of $\Delta=200$.

Since star formation occurs at the high end of the density distribution, we take the star formation density threshold as the limiting factor that defines our mass resolution. Let us assume for sake of simplicity that each halo is a pure isothermal sphere. The gas density profile is given by

$\rho=\frac{\Delta}{3} \bar{\rho}\left(\frac{r}{R_{200}}\right)^{-2}$.

The radius above which star formation occurs is given by $\rho(r)>\rho_{0}(1+z)^{\alpha_{0}}$. If we require that within this radius, we have at least 10 dark matter particles of mass $m_{\mathrm{p}}$, we obtain the minimal halo mass as

$M_{\text {resol }}=10\left[\frac{\rho_{0}(1+z)^{\alpha_{0}}}{\Delta / 3 \bar{\rho}(z)}\right]^{1 / 2} m_{\mathrm{p}}$

One clearly sees that the higher the density threshold for star formation, the larger the minimal mass will be. For star formation density thresholds constant in comoving units $\left(\alpha_{0}=3\right)$, this mass scale is a constant in time. This is the case for the AMR simulations presented here, for which, using Eq. (37), we obtain $M_{\text {resol }} \simeq 400 m_{\mathrm{p}}$. On the other hand, for star formation density threshold constant in physical units, the mass resolution scales as $(1+z)^{-1.5}$. SPH simulations presented in Springel \& Hernquist (2003b) were based on this second approach. The mass resolution we obtain in this case is
$M_{\text {resol }} \simeq 1000(1+z)^{-1.5} m_{\mathrm{p}}$. At high redshift, $z \simeq 20$, the corresponding mass resolution can be as low as $M_{\text {resol }} \simeq 10 m_{\mathrm{p}}$.

Simulated halos with mass lower than $M_{\text {resol }}$ will not be able to form stars or, equivalently, condensed cool gas. Therefore, they will be part of the simulated diffuse background. This new mass scale is a pure numerical artifact, that strongly affects our results. We take this mass scale into account in our analytical model by setting the Minimal Mass for star-forming halos $M_{\min }$ as the maximum between the true physical Minimal Mass and $M_{\text {resol }}$. As we will see later in this section, this trick is a very powerful tool to account for finite resolution effect in the simulation, and to assess the convergence properties of our numerical results.

\subsection{Halo star formation time scale}

The methodology we use in this paper is to describe star formation on a halo by halo basis. We completely discard the detailed modeling of exponential gas discs and nuclear bursts. This is usually performed in semi-analytical models of galaxy formation. In our AMR simulations, we do however have a higher level of complexity than in the analytical model. Visual inspection of density maps shows the presence of gas discs in centrifugal equilibrium, as well as several small satellites orbiting a central galaxy (see Fig. 1). We are aware of the fact that many physical ingredients are probably missing in our current numerical solution of galaxy formation. Nevertheless, we need to establish the link between our analytical model and our numerical implementation of star formation.

Since star formation in the code is based on a Schmidt law (see Eq. (1)), we can compute the instantaneous star formation rate in any halo by integrating over the entire cold gas present in that particular halo.

$\dot{M}_{*}=M_{\text {cold }} \int_{\rho_{\mathrm{t}}}^{\infty} \frac{\mu(\rho) \mathrm{d} \rho}{t_{*}(\rho)}$,

where $\rho_{\mathrm{t}}=\rho_{0}(1+z)^{\alpha_{0}}$ is the star formation density threshold and $\mu(\rho)$ is the mass fraction of cold gas with density $\rho$. The exact form of the cold gas distribution function $\mu$ is beyond the scope of this paper. It is likely to be determined by the global surface density as well as the small scale turbulence inside rotating discs. We will make here the very crude approximation that $\mu$ is self-similar in the variable $\rho / \rho_{\mathrm{t}}$, so we can simplify the last equation further into

$\dot{M}_{*}=\frac{M_{\text {cold }}}{t_{*}\left(\rho_{\mathrm{t}}\right)} F(\mu)$,

where $F(\mu)$ is a dimensionless "shape factor" that depends on the exact form of the cold gas density distribution

$F(\mu)=\int_{1}^{\infty} \mu(x) x^{1 / 2} \mathrm{~d} x$

We are now in a position to make a direct link between our analytical model and numerical simulations. We recognize in the last equation the halo star formation rate as defined in Sect. 3.5, with star formation parameters given by $\alpha_{*}=\alpha_{0}$ and $t_{*}=t_{0} / F(\mu)$. The shape factor $F$ has the effect of reducing 
the effective halo star formation time scale, relative to the reference time $t_{0}$. Indeed, if very high density gas is present, the star formation rate is likely to increase accordingly.

Since we can't predict the value of this shape factor, we have to measure it directly in the simulations. We plot in Fig. 9 the halo star formation rate, in units of $M_{\text {cold }} / t_{*}\left(\rho_{\mathrm{t}}\right)$ (see Eq. (39)), and averaged over halos of similar mass. This should be equal to the shape factor $F(\mu)$. For 3 different box sizes and at 3 different redshifts, this factor is not exactly a constant, although it varies slowly with mass. This illustrates that our approach is only a first order approximation of our simulation results. Nevertheless, we approximate this by taking $F(\mu) \simeq 3$, as suggested by the dashed line in Fig. 9. This specifies how star formation in the simulations and star formation in the model are connected to each other.

\subsection{Halo orbital decay time scale}

The only unknown parameter in our analytical model is the orbital decay time scale of infalling gas clumps (see Appendix C), before they reach the high-density disc in the halo center. When cooling is very fast, for halos with Virial temperature $T_{\min }<T_{200}<T_{\max }$, we have assumed that the accretion rate into the disc is controlled by the orbital time scale of infalling satellites. Computing this time scale is beyond the scope of this paper. It is probably determined by details in the gravitational dynamics and satellite dynamical friction. These aspects are all key ingredients of semi-analytical models of galaxy formation.

In order to determine this orbital decay time scale, we perform again a direct analysis of our numerical simulations. Let us consider the case of very fast star formation $t_{0}=3 \mathrm{Gyr}$ and $\alpha_{0}=3$. In this case, the halo star formation rate is almost equal (within 10\%) to the halo cooling rate. This can be later confirmed by the analytical model. We plot in Fig. 9 the average star formation rate of halos within different mass range, in units of $M_{\mathrm{hot}} R_{200} / V_{200}$. In our framework, this quantity is exactly equal to the ratio $\left(R_{200} / V_{200}\right) / t_{\text {orb }}$. Here again, this ratio is not perfectly a constant, illustrating the fact that our model is only a first order approximation, but for the 3 different box sizes and at 3 different redshifts, the curve exhibits a plateau around $t_{\text {orb }} \simeq 3 R_{200} / V_{200}$. We take this value as our canonical value in the analytical model.

\subsection{Global baryon budget}

We now present in greater details our simulation results, starting with the baryon history for the universe as a whole. Fig. 8 shows the baryon history in our highest resolution run L10N512S30. The run parameters correspond to a low efficiency star formation model. Each phase is defined by well structured limits in the $\rho-T$ diagram, as shown in Sect. 2.3. The various symbols in Fig. 8 refer to baryon fractions in different snapshots of the simulation, while lines refer to the analytical model predictions, with $M_{\text {resol }} \simeq 2 \times 10^{8} h^{-1} M_{\odot}$, as given by Eq. (37). The other parameters of the model are set to their standard values $\left(F(\mu)=3\right.$ and $\left.R_{\text {orb }}=3 R_{200}\right)$. The agreement

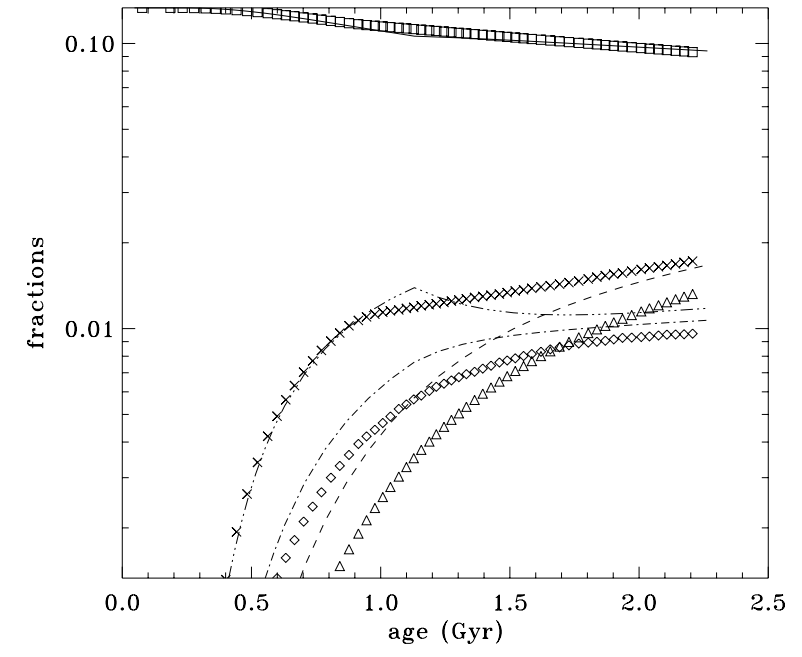

Fig. 8. Time evolution of the various baryon phases in our highest resolution run L10N512S30. Symbols are mass fractions measured in the simulation (squares: diffuse background, crosses: hot gas, diamonds: cold gas and triangles: stars). Lines are the predictions of our analytical model with $M_{\text {resol }} \simeq 2 \times 10^{8} h^{-1} M_{\odot}$ (solid: diffuse background, dot-dot-dashed: hot gas, dot-dashed: cold gas and dashed: stars).

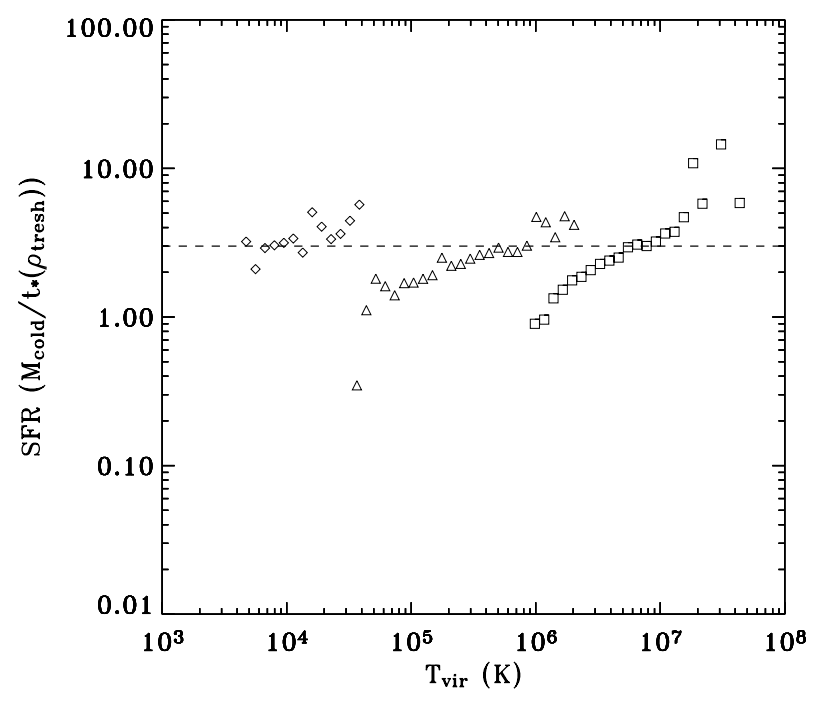

Fig. 9. Average star formation rate of simulated halos in various Virial temperature bins, in units of $M_{\text {cold }} / t_{*}\left(\rho_{t}\right)$. In our framework, this is a direct measure of the "shape factor" $F(\mu)$ of the underlying cold gas density distribution. Numerical data suggest a constant value represented here as the dashed line $F(\mu) \simeq 3$. Diamonds are for run $\mathrm{L} 1 \mathrm{~N} 256 \mathrm{~S} 30$ at $z=5.5$, triangles for run $\mathrm{L} 10 \mathrm{~N} 256 \mathrm{~S} 30$ at $z=2.5$ and squares for run L100N256S30 at $z=0$.

between the simulation and the model is very good (within a factor of 2), given the simplicity of the latter.

We now examine more closely the global star formation rate as a function of redshift, measured in all our simulations, and compare our various results to the analytical model. This quantity is a key prediction of the hierarchical model of galaxy formation. It translates more or less directly into galaxy colors and luminosities, and provides a stringent test of the current cosmological theory. As star particles are created during the course of a simulation, we keep track of their birth epoch. It is 


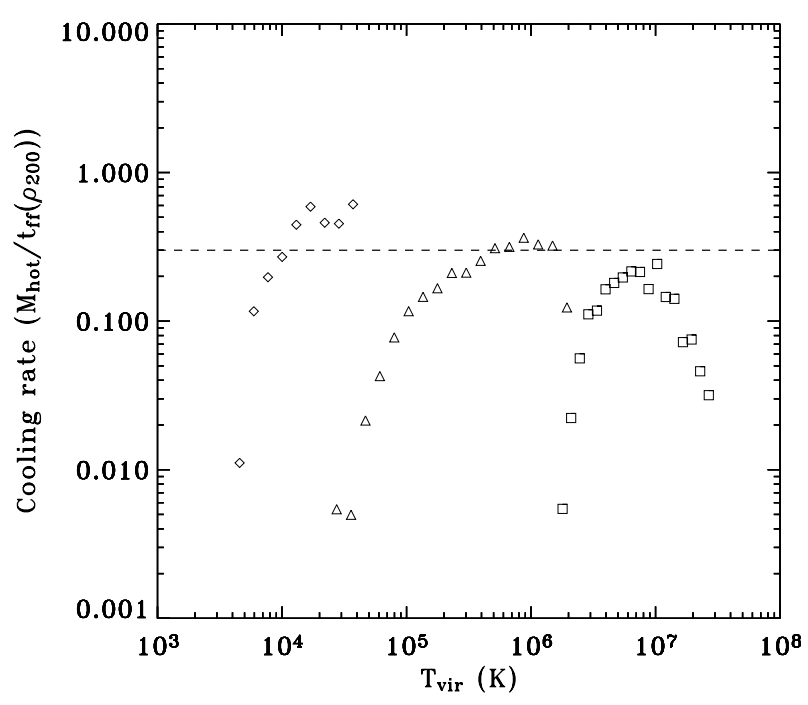

Fig. 10. Average star formation rate of simulated halos in various Virial temperature bins, in units of $M_{\mathrm{hot}} R_{200} / V_{200}$ for the high efficiency runs. This is a direct measure of the cooling rate of hot gas into dense cold discs. At high temperature $\left(T>10^{7} \mathrm{~K}\right)$, we observe a sudden drop due to inefficient Bremstrhalung cooling. At lower temperatures, the cooling rate has a plateau around $1 / 3$. In our framework, this suggests an orbital decay time scale $t_{\text {orb }} \simeq 3 R_{200} / V_{200}$ for infalling gas clumps. Diamonds are for run L1N256S3 at $z=5.5$, triangles for run L10N256S3 at $z=2.5$ and squares for run L100N256S3 at $z=2.5$ also.

straightforward to compute, using the last output only, the star formation epoch histogram (history).

Figure 11 shows this global star formation history for our "convergence study" simulation suite: L10N64S30, L10N128S30, L10N256S30 and L10N512S30. Numerical results are shown as symbols, while analytical predictions are shown as lines. The analytical model predictions are computed with a Minimal Mass corresponding to the mass resolution of each run, as given by Eq. (37). Since our star formation recipe is based on a constant overdensity threshold, the mass resolution is $M_{\text {resol }} \simeq 400 m_{\mathrm{p}}$. The solid line stands for the analytical prediction, without any finite resolution effects $\left(M_{\text {resol }}=0\right)$. This gives an indication on how our results have converged to the "true" star formation history in this particular model.

Around $z \simeq 3-5$, our highest resolution run L10N512S30 is close to the correct value. At very high redshift however, the star formation rate is lower than the expected value by a factor of ten. The mass resolution $M_{\text {resol }}$ is indeed significantly higher than $M_{\min }$ at redshift $z>10$ : this explains the origin of this discrepancy. As illustrated by the Fig. 3, low resolution runs do miss the formation of dwarf galaxies that contribute significantly to the global star formation history.

This first series of simulations was performed for a box length $L=10 h^{-1} \mathrm{Mpc}$. By $z \simeq 3$, the non-linear scale becomes comparable to the box size. We have to stop the simulations, as our realizations are not representative of the universe as a whole anymore.

Our second series of simulations was designed to explore larger $\left(L=100 \mathrm{~h}^{-1} \mathrm{Mpc}\right)$ and smaller $\left(L=1 \mathrm{~h}^{-1} \mathrm{Mpc}\right)$ scales, as well as another, more efficient, star formation scenario with $t_{0}=3 \mathrm{Gyr}$. The corresponding Schmidt law is therefore ten times more effective than in the first case. The resolution was fixed to $256^{3}$ particles. All six results are shown in Fig. 11 with symbols, while the corresponding analytical predictions are overploted with lines. Note that the smaller box ( $L=1 h^{-1} \mathrm{Mpc}$ ) has a mass resolution smaller than $M_{\min }$. It has therefore converged to the "true" star formation history. The analytical model is indeed in good agreement with our numerical results. These very small scale simulations have to be stopped even earlier than the previous ones $(z \simeq 5)$. The largest box size ( $L=100 h^{-1} \mathrm{Mpc}$ ), on the other hand, is strongly affected by finite mass resolution effect. The star formation history is drastically different from the other two. Star formation begins very late, around $z \simeq 5$, and the peak value is one order of magnitude lower than the "true" star formation rate. Our analytical model again provides a good fit for numerical data, when the poor mass resolution is taken into account to define the diffuse background.

Let us now compare the two different star formation scenarios (inefficient with $t_{0}=30 \mathrm{Gyr}$ and efficient with $t_{0}=3 \mathrm{Gyr}$ ). Both star formation histories are parallel to each other, but with a factor of two difference only. For the very efficient scenario, star formation is limited by accretion and cooling, rather than by the Schmidt law. As discussed in the next section, very efficient star formation models give SFR quasi independent of the physical parameters. This is confirmed by our numerical simulations. The agreement between the simulation and the model is extremely good, except at low redshift $(z<3)$, for our large box runs, where the model significantly overestimates the star formation rate. This disagreement might be reduced by improving the analytical model, along several lines we have outlined in this paper. Note that finite volume effect might also have an additional effect on the numerical simulation predictions, but we do not try to include those subtleties in the analytical model.

From now on, we have analyzed AMR simulations for which star formation is triggered in regions where the gas density exceeds an overdensity threshold. In this case, we naturally compare our numerical results to the "accelerated efficiency" analytical model. We now test the model predictions for the "constant efficiency" analytical model, using SPH results kindly provided us by Springel \& Hernquist (2003b). Our interest in these SPH results is twofold: first, star formation is triggered in regions where the gas density exceeds a physical density threshold. This scenario, as already discussed in Sect. 4.3, corresponds to a "constant efficiency" star formation model. Second, Springel \& Hernquist (2003b) have included galactic winds in their numerical modeling, giving us the opportunity to test our simple feedback model.

In Fig. 11 are shown Springel \& Hernquist (2003b) results when galactic winds are turned off. The only difference with our simulations, apart from the overall numerical techniques, comes from the star formation details. They have considered the following parameters $t_{0}=2.1 \mathrm{Gyr}, n_{0}=0.1 \mathrm{~cm}^{-3}$ and $\alpha_{0}=0$. For the analytical model, we use the same "shape factor" $F(\mu)=3$, as determined earlier using our AMR results. This translates into the following parameters for the analytical model: $t_{*}=0.7 \mathrm{Gyr}$ and $\alpha_{*}=0$. Taking into account the finite mass resolution (using Eq. (11)), we can now compare our 

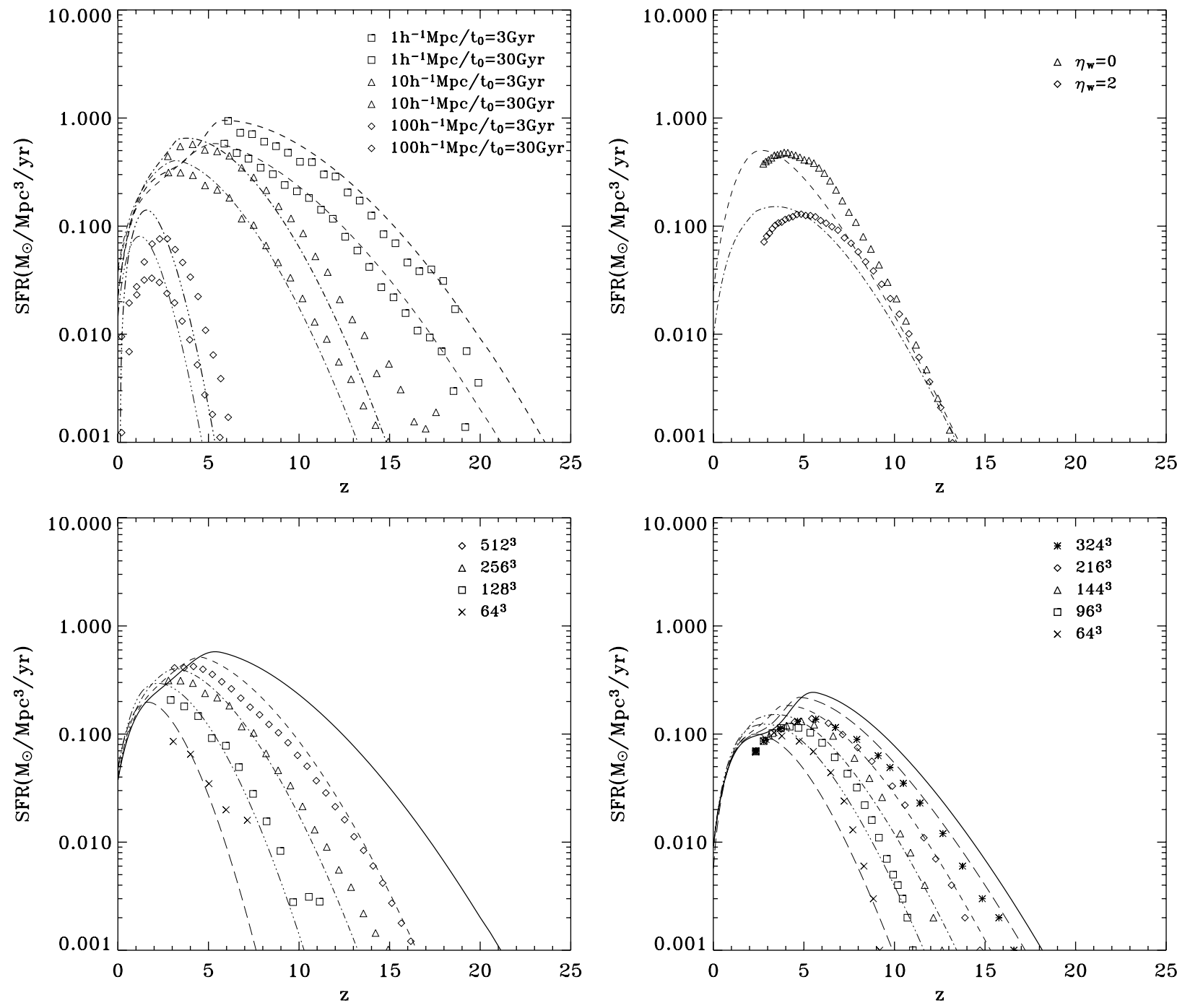

Fig. 11. Global comoving star formation rate as a function of redshift. In each plot, symbols are for numerical simulations, while lines are for our corresponding analytical prediction. Upper left plot: high efficiency series and low efficiency series with run L1N256S3 (black squares), run L1N256S30 (grey squares), run L10N256S3 (black triangles), run L10N256S30 (grey triangles), run L100N256S3 (black diamonds) and run L100N256S30 (grey diamonds). Lower left plot: convergence study series with run L10N512S30 (diamonds), run L10N256S30 (triangles), run L10N128S30 (squares) and run L10N64S30 (crosses). Upper right plot: Springel E Hernquist (2003b) simulations with run 03 without winds (triangles) and Q3 including winds (diamonds). Lower right plot: Springel \& Hernquist (2003b) simulations including winds with run Q5 (stars), run Q4 (diamonds), run Q3 (triangles), run Q2 (squares) and run Q1 (crosses). The solid line is the "fully converged" model prediction.

analytical prediction to SPH results. The agreement is clearly very good: this is rather encouraging for our model, since our main unknown parameter, the mean orbital length, was kept fixed to its canonical value $R_{\text {orb }}=3 R_{200}$, calibrated on our AMR results.

We now analyze SPH results when galactic winds are turned on. Remember that winds are assumed to eject cold gas from the rotating discs at a typical wind velocity $u_{\mathrm{w}} \simeq$ $250-500 \mathrm{~km} \mathrm{~s}^{-1}$ and with an efficiency parameterized by $\eta_{\mathrm{w}}$. This approach, directly inspired by Springel \& Hernquist (2003b), allows a straightforward comparison between our analytical model and SPH results with winds. This comparison is shown in Fig. 11. The analytical model parameters were set to $T_{\mathrm{w}}=2 \times 10^{6} \mathrm{~K}$ and $\eta_{\mathrm{w}}=3$. The Virial temperature $T_{\mathrm{w}}$ (below which winds are unbound from their parent halo) corresponds closely to the wind velocity $u_{\mathrm{w}} \simeq 500 \mathrm{~km} \mathrm{~s}^{-1}$ used by Springel \& Hernquist (2003b) in their SPH simulation (see Eq. (31)). The wind efficiency parameter, however, was chosen $50 \%$ higher than the value $\eta_{\mathrm{w}}=2$ used in the SPH simulation. As suggested by Springel \& Hernquist (2003b), the global wind efficiency (at the halo scale) is higher than the local wind efficiency (at the star-forming regions scale) due to gas entrainement: additional cold gas, lying outside star-forming regions from which winds originate, can be expelled by the ram pressure of the outflow. Figure 11 shows again a good agreement between SPH simulation results and our analytical model.

We consider now SPH results from the "Q series" in Springel \& Hernquist (2003b) paper, in order to study finite mass resolution effects. Wind parameters are fixed to $T_{\mathrm{w}}=$ $2 \times 10^{6} \mathrm{~K}$ and $\eta_{\mathrm{w}}=3$. Note that in this case, due to a star formation strategy based on a constant physical density, $M_{\text {resol }}$ is now varying with time $M_{\text {resol }} \simeq 1000(1+z)^{-1.5} m_{\mathrm{p}}$. In Fig. 11 
are plotted Springel \& Hernquist (2003b) simulation results together with our model predictions, when mass resolution effects are taken into account. At high redshift, we recover the correct convergence towards the asymptotic $\left(M_{\text {resol }}=0\right)$ converged curve. At intermediate redshift, however, the agreement is getting worse, although both curves remain close to each other within a factor of 2. One possible reason is that Eq. (37) is not accurate enough to estimate the effective halo mass resolution of SPH simulations, especially in presence of winds.

We conclude that the global baryon history we obtain in numerical simulations is correctly described by our analytical model, when finite mass resolution effects are taken into account. This was tested for 2 different numerical technics (AMR and SPH), two different star formation scenarios ("constant" and "accelerated" efficiency), with and without galactic winds. The main effect of insufficient mass resolution is to artificially decrease the average age of stars in the universe and to lower the star formation rate at peak value.

\subsection{Halo baryon budget}

We now present our simulation results concerning the baryon budget in individual halos. Halos are detected in the simulations according to the method explained in Sect. 4.1. The Extended Press \& Schechter (EPS) theory gives us the opportunity to apply our analytical method to an "average" halo of mass $M_{0}$ at redshift $z_{0}$. The model predictions can then be compared to the average baryon fractions (in each of the 4 different baryon phases), the average being taken over all halos in a given mass range, centered around $M_{0}$.

Using all star particles found within $R_{200}$, we compute the star formation history within each halo. We then compute the star formation rate as explained in Sect. 4.1. Figure 12 shows our various numerical results, including the SPH simulations with winds provided to us by Springel \& Hernquist (2003b), together with the analytical model predictions. We have plotted the halo star formation rate as a function of the halo temperature $T_{200}$, in units of $M_{200} / t$. This definition corresponds closely to the "specific star formation rate" defined by Springel \& Hernquist (2003b).

Each curve shows a sharp cut-off at the low mass end, corresponding to the Minimal Mass for each run. The "convergence study" series clearly illustrates that this Minimal Mass is in fact equal to the mass resolution $M_{\text {resol, except for our }}$ smallest box size. Another non trivial effect of finite mass resolution is to increase the halo star formation rate. This is due to a higher Cosmic Accretion Rate $\left(\dot{f}_{\text {acc }} \propto \sigma\left(M_{\text {min }}\right)^{-1}\right.$, see Eq. (19)), as $M_{\min }$ is increased. Our analytical model predictions are in good agreement with the halo star formation rates, even when winds are present, as soon as finite mass resolution is explicitly accounted for in the model. The role of winds is to remove cold gas from small mass halos $T_{200}<2 \times 10^{6} \mathrm{~K}$, so that the halo star formation rate decreases accordingly.

When we compare the high star formation efficiency series with $t_{0}=3$ Gyr with the low star formation efficiency series with $t_{0}=30 \mathrm{Gyr}$, we see that the former has a higher halo star formation rate at high redshift, but a much lower halo star formation rate at low redshift, as cold gas is almost completely consumed. This complex behavior is well reproduced by the analytical model. Both series show a sharp decline of the halo star formation rate at the high mass end: the cooling efficiency decreases for high mass halos and the mass accretion rate on cold discs vanishes. Note that we assumed here a zero metallicity plasma. Gas cooling is likely to be more efficient around $T_{200} \simeq 10^{7} \mathrm{~K}$ if metals are present.

We now present the baryon budget inside dark matter halos, as a function of the halo Virial temperature. In each halo, we compute the total baryon fraction, which can be further decomposed into hot gas, cold gas and stars, using the definitions of Sects. 2.3 and 4.1. Figure 13 shows this halo baryon budget for the "high efficiency" simulation series, Fig. 14 for the "low efficiency" simulation series and Fig. 15 for the "convergence study" simulation series.

In each case, there is good agreement between numerical and analytical results. The total baryon fraction is close to the average value $f_{\mathrm{b}}$ for halos more massive than $M_{\min }$ and vanishes for smaller halos. Note that the total baryon fraction is actually slightly above the universal value for massive halos. Dissipative collapse of baryons condenses more mass than collisionless collapse of dark matter. The analytical model predicts a sharp transition for $M<M_{\text {resol }}$ where the total baryon fraction vanishes. In the simulations, this transition is much smoother, but its location is correctly predicted around $M_{\text {resol }}$. On the other hand, when the mass resolution is small enough, the low mass end of the analytical curve has also a smooth transition. This comes from halos whose mass was greater than $M_{\min }$ at early time, but as reionization heats up the background temperature, these same halos end up with a mass smaller than $M_{\min }$. The total baryon fraction at the final redshift is therefore smaller than the universal value but greater than zero.

The hot gas fraction is also strongly affected by resolution effect. As we resolve smaller and smaller mass progenitors, more and more gas is condensed into cold discs and eventually stars. Figure 15 shows the hot gas fraction for the "convergence study" series and one clearly sees that our poorest resolution run overestimates the hot gas fraction by a factor of 3 . As a consequence, the cold gas and star mass fraction gradually increase as the mass resolution is improved. The curve showing the cold gas fraction as a function of Virial temperature is very similar to the halo star formation rate, as expected from our analytical model, although they have been computed completely differently in the simulation analysis. In the "high efficiency" series, our largest box run L100N256S3 has almost entirely consumed the cold gas of the most massive halos, where cooling has virtually stopped (see Fig. 13). The corresponding run with a "low efficiency" star formation scenario still shows some surviving cold gas at the high mass end of the halo distribution.

We conclude at the end of this section that our analytical model has proven to successfully reproduce the complex behavior observed in our simulations and in simulations of Springel \& Hernquist (2003b) (with winds) if finite resolution effects are taken into account. We note however a slight tendency of the model to overestimate the star formation rate at low redshift. The baryon budget was analyzed in great detail 

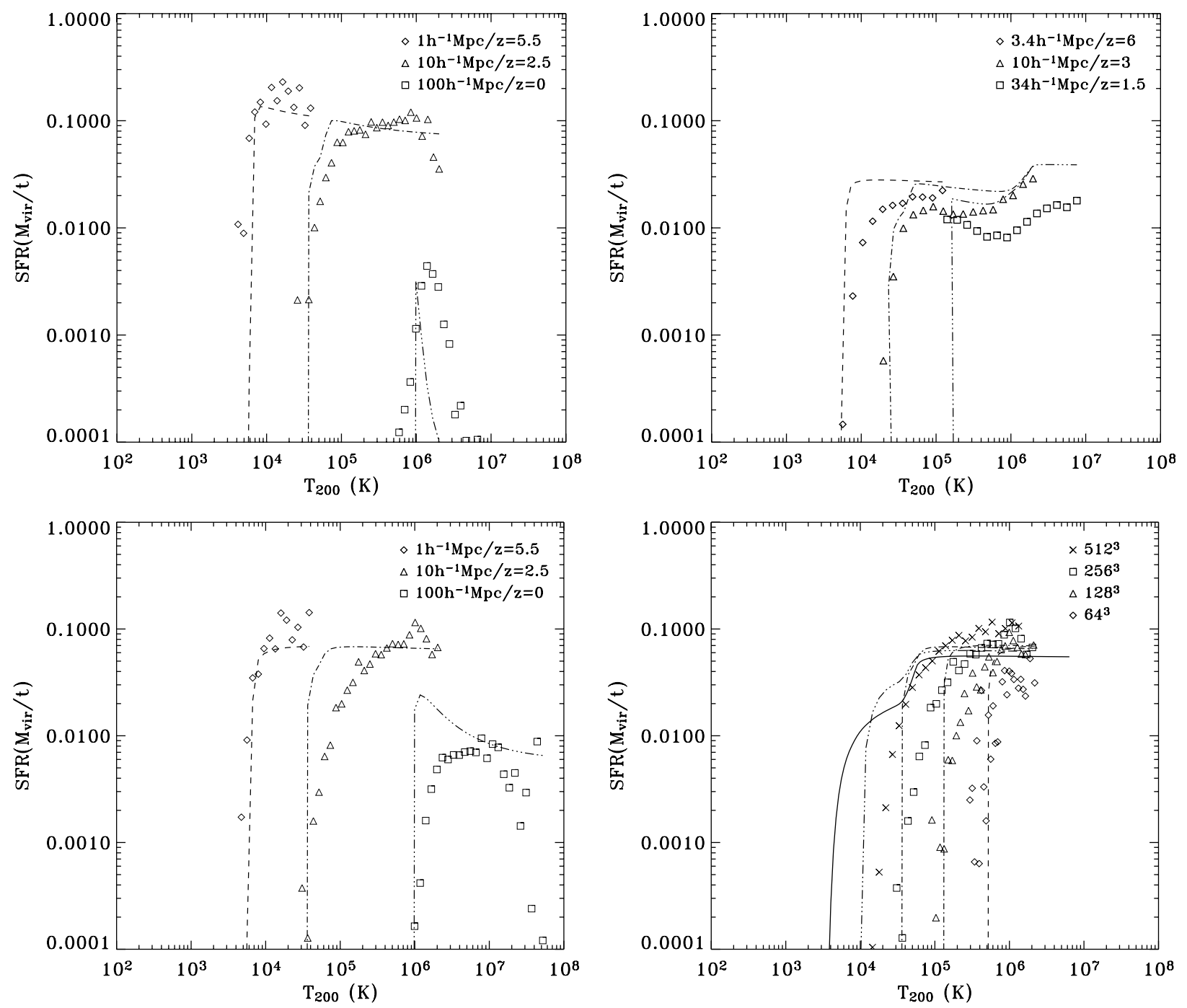

Fig. 12. Halo star formation rate, in units of $M_{200} / t$, as a function of the halo Virial temperature. In each plot, symbols are for numerical simulations, while lines are for our corresponding analytical prediction. Upper left plot: high efficiency series with run L1N256S3 at $z=5.5$ (diamonds), run L10N256S3 at $z=2.5$ (triangles) and run L100N256S3 at $z=0$. Lower left plot: low efficiency series with run L1N256S30 at $z=5.5$ (diamonds), run L10N256S30 at $z=2.5$ (triangles) and run L100N256S30 at $z=0$. Lower right plot: convergence study series at $z=2.8$ with run L10N64S30 (diamonds), run L10N128S30 (triangles), run L10N256S30 (squares) and run L10N512S30 (crosses). The solid line is the "fully converged" model prediction. Upper right plot: Springel $\mathcal{E}$ Hernquist (2003b) simulations including winds, with run R4 (diamonds) at $z=6$, run $\mathrm{Q} 4$ at $z=3$ (triangles) and run $\mathrm{D} 4$ at $z=1.5$ (squares).

on a halo by halo basis (and in an average sense). We emphasized the important role played by the Minimal Mass $M_{\min }$ in controlling the baryon history in each mass range. We also described how cooling and winds introduce characteristic features into the various plots showing the baryon evolution as a function of the halo Virial temperature.

\section{Observations versus model}

From now on, we assume that our analytical model gives an accurate (within a factor of 2) modeling of the baryon history in a hierarchical universe. The careful comparison to numerical simulations we performed in the previous section was a necessary step to calibrate our model and to estimate its level of accuracy. We are now in a position to use this model as a tool to analyze current observational constraints put on the baryon history in the universe.

\subsection{Model parameters study}

We briefly recall how the analytical predictions depend on the model parameters. We consider that all cosmological parameters are fixed to their "concordance" $\Lambda \mathrm{CDM}$ value, as we did throughout this paper. We also assume that the reionization temperature is given by $T_{\mathrm{r}} \simeq 6 \times 10^{3} \mathrm{~K}$. We finally use the cooling model of Sect. C, valid for a primordial, zero metallicity, $\mathrm{H}$ and He plasma and a wind velocity $u_{\mathrm{w}} \simeq 500 \mathrm{~km} \mathrm{~s}^{-1}$. We end up with 3 main parameters that we allow to vary in our model: the reionization redshift $z_{\mathrm{r}}$, the star formation time scale (or consumption time scale) $t_{*}=M_{\text {cold }} / \dot{M}_{*}$ and the wind efficiency $\eta_{\mathrm{w}}=\dot{M}_{\text {wind }} / \dot{M}_{*}$. We consider in this section only "constant 

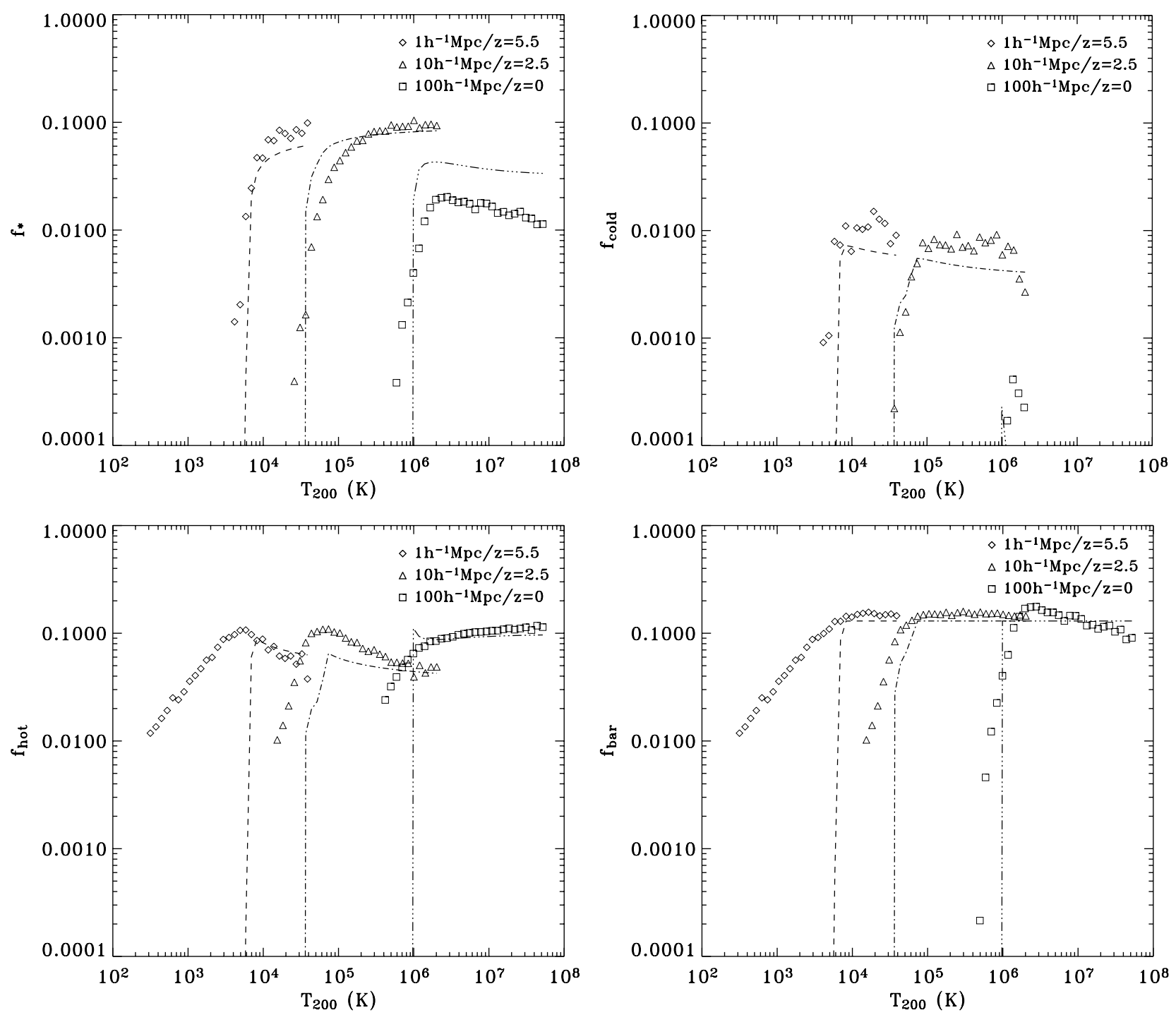

Fig. 13. Mass fraction in each baryon phase as a function of halo Virial temperature for the high efficiency simulation series. In each plot, diamonds refer to run L1N256S3 at $z=5.5$, triangles to run L10N256S3 at $z=2.5$ and squares to run L100N256S3 at $z=0$. Lines are for the corresponding analytical model. The upper left plot shows the star mass fraction; the lower left plot shows the hot gas mass fraction; the lower right plot shows the total baryon fraction and the upper right plot shows the cold gas fraction.

efficiency" star formation models $\alpha_{*}=0$. The alternate scenario with "accelerated efficiency" star formation and $\alpha_{*}=3$ gives similar, though slightly poorer, results. Using current observational constraints, it is not easy to discriminate between these two models.

We now present the model predictions for the Cosmic Star Formation Rate, for different values of $z_{\mathrm{r}}, t_{*}$ and $\eta_{\mathrm{w}}$. Figure 16 corresponds to an "infinite efficiency" star formation model $\left(t_{*}=0 \mathrm{Gyr}\right)$, for various reionization redshifts. In this extreme case, the cosmic star formation rate (CSFR) is equal to the mass accretion rate of cold discs, since star formation is instantaneous. At reionization, the background gas is promptly heated to $10^{4} \mathrm{~K}$ and the star formation rate drops. We notice that at redshifts lower than 6 , all models agree with one another, leading us to the conclusion that, as soon as reionization proceeds early enough, this parameter is in fact irrelevant to the low redshift universe.
Figure 17 shows the influence of the two remaining (and really interesting) parameters. Star formation inside cold, centrifugally supported, galactic discs acts merely as a delay with respect to diffuse gas accretion. The epoch of peak star formation is delayed from $z \simeq 4$ for $t_{*}=0$ down to $z \simeq 1$ for $t_{*}=10 \mathrm{Gyr}$. It is worth noticing that the amplitude of the global star formation rate is mainly determined by the cosmological accretion rate, and that the small scale, poorly known, physics of star formation introduces a rather small modification to the curve. When winds are included in the model, they lower significantly the amplitude of the CSFR. They also have the non trivial effect of advancing the epoch of peak star formation, from $z \simeq 1$ for $\eta_{\mathrm{w}}=0$ up to $z \simeq 3$ for $\eta_{\mathrm{w}}=10$. They play an important role at low redshift whereas at high redshift the CSFR seems independent of $\eta_{\mathrm{w}}$. Indeed, for the "constant efficiency" recipe the star formation rate at high redshift is so small compared to the accretion rate that winds (which are 

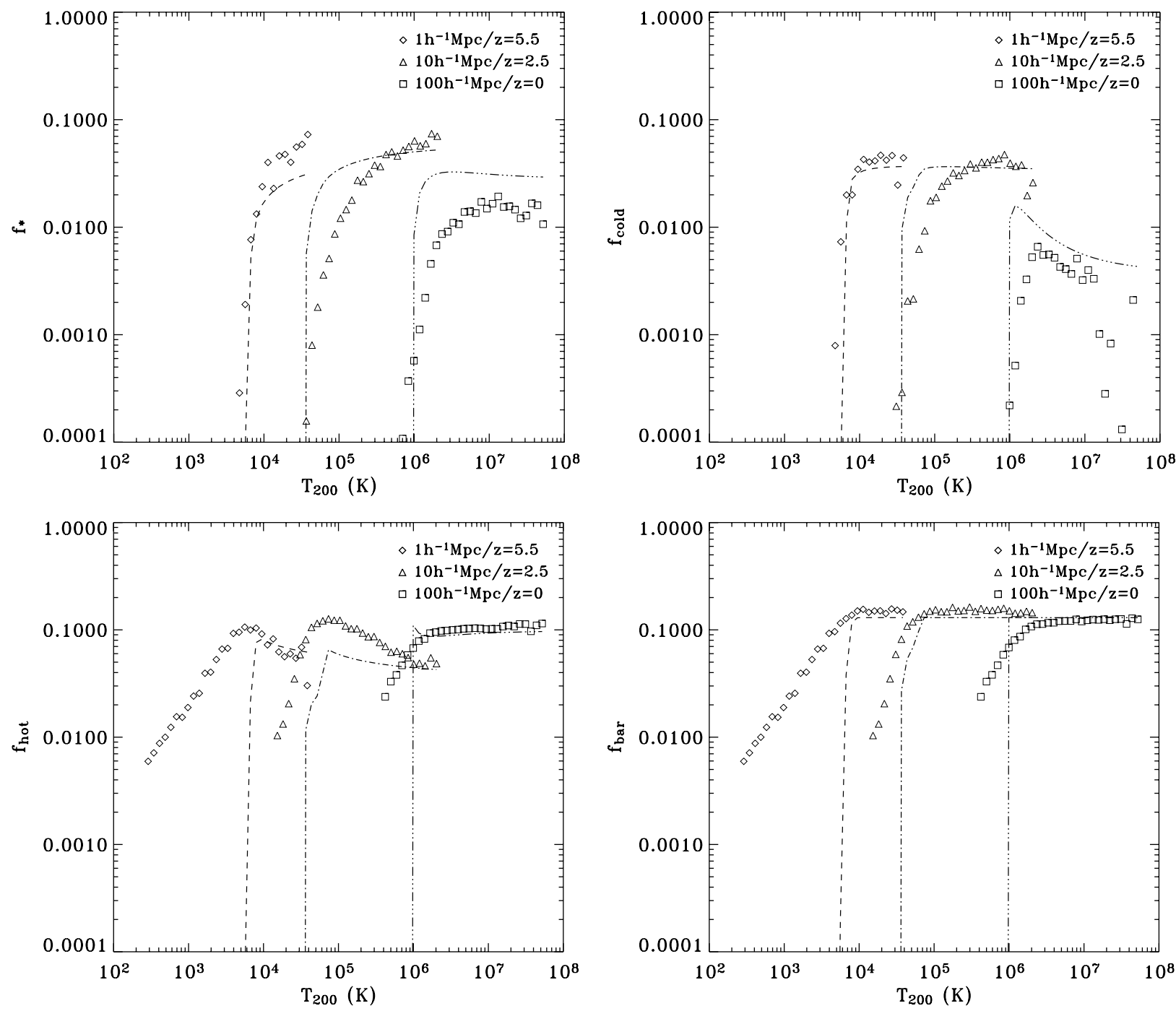

Fig. 14. Mass fraction in each baryon phase as a function of halo Virial temperature for the low efficiency simulation series. In each plot, diamonds refer to run L1N256S30 at $z=5.5$, triangles to run L10N256S30 at $z=2.5$ and squares to run L100N256S30 at $z=0$. Lines are for the corresponding analytical model. The upper left plot shows the star mass fraction; the lower left plot shows the hot gas mass fraction; the lower right plot shows the total baryon fraction and the upper right plot shows the cold gas fraction.

proportional to the star formation rate) do not affect the amount of cold gas.

Computing the baryon history using our analytical model is a very fast operation. This gives us the opportunity to compute various observational quantities for a grid of model parameters. Using various observations, we will now try to constrain our parameter space and shed light on these 2 important galaxy formation ingredients: $t_{*}$ and $\eta_{\mathrm{w}}$.

\subsection{Cosmic star formation rate}

One of the main goals of this paper is to compute the star formation history in a hierarchical universe. We will now compare the model predictions to the observed star formation rate. Figure 18 shows the observational data points, usually referred to as the "Madau plot", compiled by Elbaz (2005) and uniformally corrected from cosmological distances, incompletness and dust absorption. Original data points came from Hughes et al. (1998); Steidel et al. (1999); Flores et al. (1999); Glazebrook et al. (1999); Yan et al. (1999); Massarotti et al. (2001); Giavalisco et al. (2004). These data points indicates an epoch of peak star formation rate at $z \simeq 2$, followed by a rapid fall off (by a factor around ten) between $z=1$ and $z=0$.

The shape and the normalization of the Madau plot are well reproduced by the parameter choice $t_{*} \simeq 1.5 \mathrm{Gyr}$ and $\eta_{\mathrm{w}} \simeq 1$. It is worth noticing that this star formation time scale corresponds roughly to the value one can infer from the consumption time scale in local galaxies (Kennicutt et al. 1994; Kennicutt 1998). The wind efficiency we obtain from this fitting exercise is close to the value inferred from $\mathrm{H}_{\alpha}$ observations of high-SFR galaxies (Martin 1999). Galactic winds are required in order to reproduce the rapid fall off of the star formation rate at low redshift.

The observational constraints on our two main star formation parameters are summarized in the upper right plot of Fig. 18, which represents contours of constant star formation rates at $z=0$ and $z=3$ in the $\eta_{\mathrm{w}}-t_{*}$ plane. The observed 

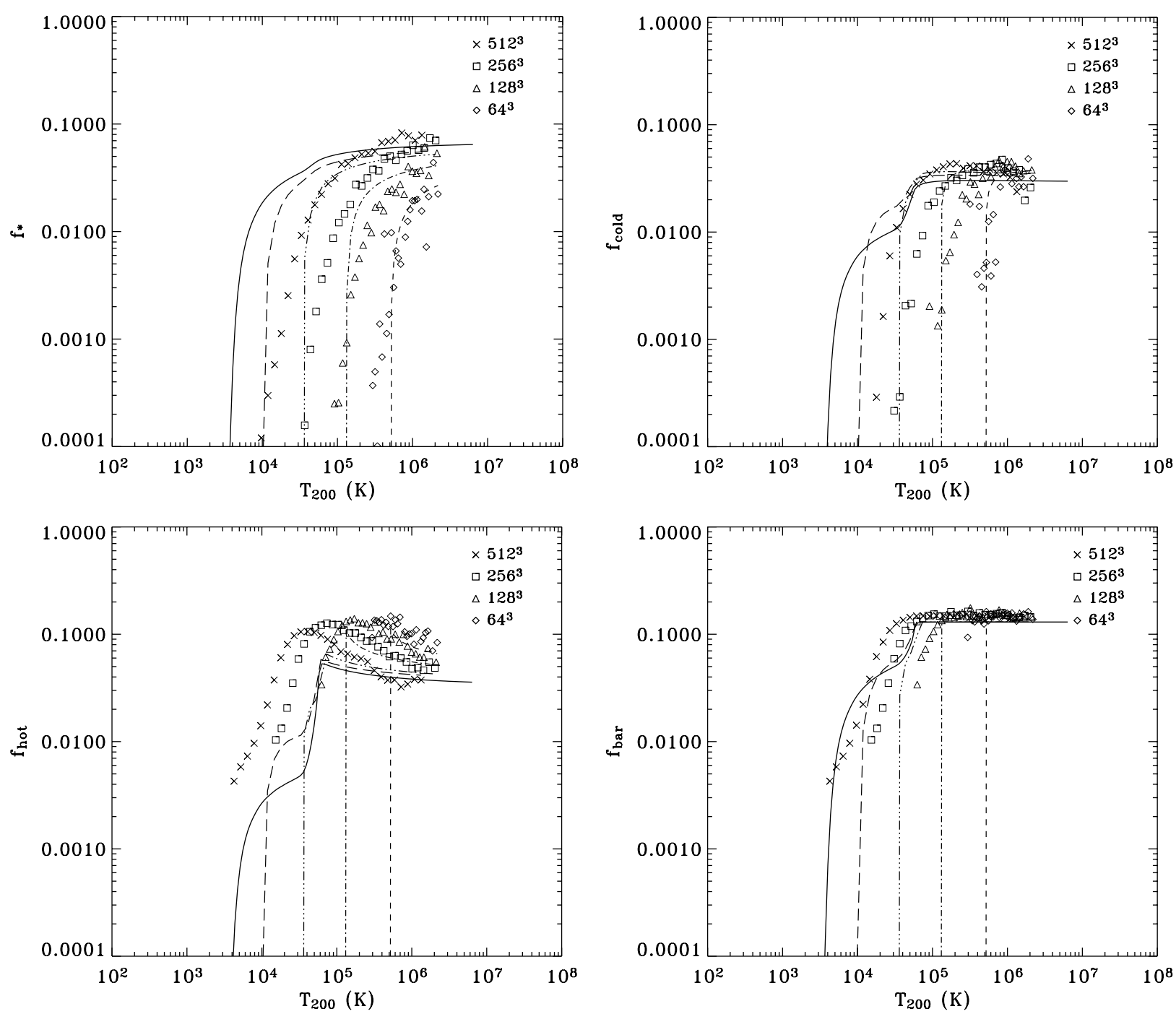

Fig. 15. Mass fraction in each baryon phase as a function of halo Virial temperature for the convergence study simulation series at $z=2.8$. In each plot, diamonds refer to run L10N64S30, triangles to run L10N128S30, squares to run L10N256S30 and crosses to run L10N512S30. Lines are for the corresponding analytical model. The upper left plot shows the star mass fraction; the lower left plot shows the hot gas mass fraction; the lower right plot shows the total baryon fraction and the upper right plot shows the cold gas fraction. The solid lines are the "fully converged" model predictions.

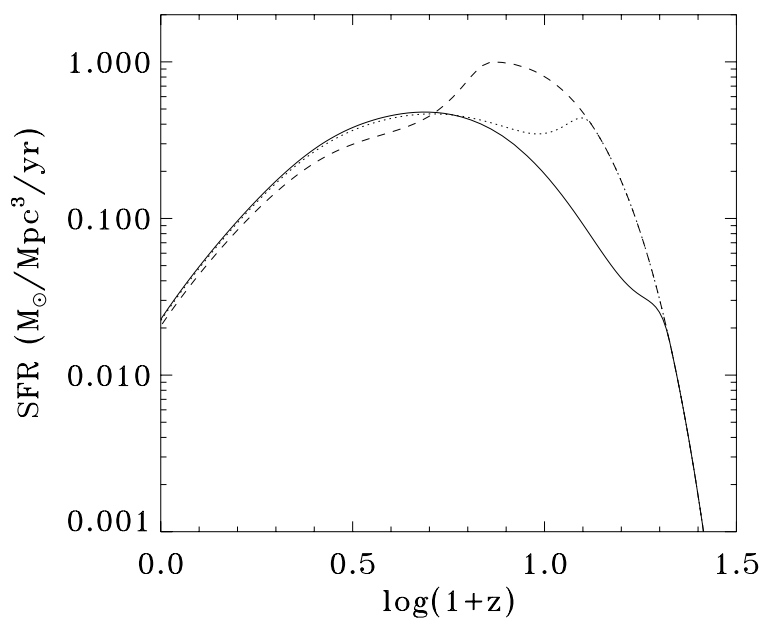

Fig. 16. Cosmic Star Formation Rate for different model parameters: $t_{*}=0$ and $\eta_{\mathrm{w}}=0, z_{\mathrm{r}}=20$ (solid line), $z_{\mathrm{r}}=12$ (dotted line), $z_{\mathrm{r}}=6$ (dashed line). central value is shown as the bold lines. The two other contours correspond to a SFR value, a factor of 2 higher and lower than the central value. As we see, the main constraint given by the observed SFR is that $t_{*}$ must be lower than 5 Gyr. If not, the SFR will be underestimate at high redshift by a factor more than 2. The contours also indicate that winds are required to reproduce the low SFR observed at low redshift. More qualitatively, winds are also required to prevent "the overcooling problem" and to remove baryons from the cold gas phase.

\subsection{Cosmic stellar density}

A complementary method to investigate the history of the baryon assembly in the universe is to observe the evolution of the global stellar mass density $\Omega_{*}(z)$. This is an independent way of constraining the model, since the SFR is related to young stars, while cosmic stellar observations focus on old, red and low mass stars. The middle left plot of Fig. 18 shows 

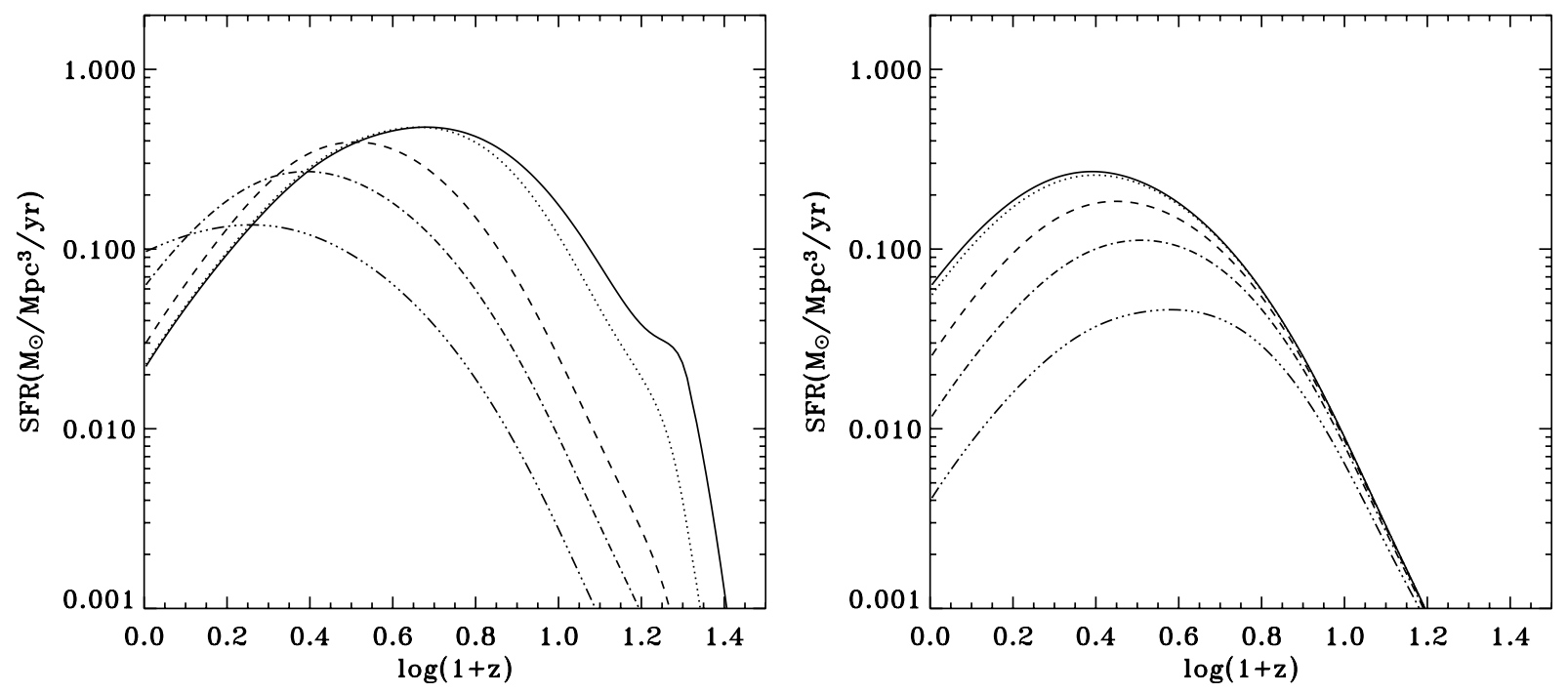

Fig. 17. Cosmic Star Formation Rate for different model parameters. Left plot: $z_{\mathrm{r}}=20$ and $\eta_{\mathrm{w}}=0, t_{*}=0$ (solid line), $t_{*}=0.1 \mathrm{Gyr}$ (dotted line), $t_{*}=1 \mathrm{Gyr}$ (dashed line), $t_{*}=3 \mathrm{Gyr}$ (dot-dashed line), $t_{*}=10 \mathrm{Gyr}$ (dot-dot-dashed line). Right plot: $z_{\mathrm{r}}=20$ and $t_{*}=3 \mathrm{Gyr}, \eta_{\mathrm{w}}=0$ (solid line), $\eta_{\mathrm{w}}=0.1$ (dotted line), $\eta_{\mathrm{w}}=1$ (dashed line), $\eta_{\mathrm{w}}=3$ (dot-dashed line), $\eta_{\mathrm{w}}=10$ (dot-dot-dashed line).

the observational data points of $\Omega_{*}$ compiled by Dickinson et al. (2003) from various near-infrared and optical observations (Cole et al. 2001; Brinchmann \& Ellis 2000; Cohen 2002; Dickinson et al. 2003).

In order to perform an accurate comparison, we need to take into account the death of short-lived stars during the history of the universe. Since most of the stars are formed when the universe is a few Gyr young, this effect is likely to be important. We use here the stellar Initial Mass Function (IMF) proposed by Kroupa et al. (1993) in order to estimate the amount of stars still alive at a given redshift. The corresponding predicted stellar density is plotted in the middle left plot of Fig. 18. Our best fit model for this observation is now $t_{*}=10 \mathrm{Gyr}$ and $\eta_{\mathrm{w}}=0.5$, in complete disagreement with our best-fit model for the Cosmic SFR. This rather surprising result is due entirely to the high redshift observations of $\Omega_{*}$. On the other hand, low redshift observations are compatible with the SFR constraints.

This puzzle was identified as a possible "missing galaxy problem" (Nagamine et al. 2004). This indicates that, if the IMF remains universal, the star formation rate should fall off at high redshift more strongly than in Fig. 18. In the model, this can be obtained for $t_{*}=10 \mathrm{Gyr}$, but then, as it can be seen in Fig. 17, the SFR is too high at low redshift. The possible inconsistency between the observed SFR and the observed stellar density is discussed in detail in Dickinson et al. (2003). The mass-luminosity relation might be poorly estimated at high redshift because the galaxy luminosity function is not well constrained. Another solution is to invoke an evolving IMF, with more high mass stars at high redshift. Finally, the last solution is to invoke a new physical process, not included in the model, which inhibits star formation at high redshift.

\subsection{Extragalactic background light}

Another strong observational constraint is the Extragalactic Background Light (EBL), integrated from the UV to the IR.
The EBL is an estimate of the total amount of energy emitted by stars and AGN in the history of the universe. Consequently, the cumulative luminosity of all stars created by the model cannot exceed the value of this background. Madau \& Pozzetti (2000) compute the value of the observed EBL, integrating from 0.2 to $2000 \mu \mathrm{m}$, and found $I_{\mathrm{EBL}}=55 \mathrm{nW} / \mathrm{m}^{2} / \mathrm{sr}$. Following the method presented in Madau \& Pozzetti (2000), we compute the EBL corresponding to our canonical model

$I_{\mathrm{EBL}}=\frac{c}{4 \pi} \int_{0}^{+\infty} \frac{\rho_{\mathrm{bol}}(t)}{1+z}\left|\frac{\mathrm{d} t}{\mathrm{~d} z}\right| \mathrm{d} z$,

with the bolometric emissivity at time $\mathrm{t}$

$\rho_{\mathrm{bol}}(t)=\int_{0}^{t} L(\tau) \dot{\rho}_{*}(t-\tau) \mathrm{d} \tau$

In this expression, $L(\tau)$ is the bolometric luminosity of a single stellar cluster of unit mass, as a function of the cluster age $\tau$. We use for $L(\tau)$ the analytical approximation given by Madau \& Pozzetti (2000) for a solar metallicity stellar population. We find for our canonical model $I_{\mathrm{EBL}} \simeq 100 \mathrm{nW} / \mathrm{m}^{2} / \mathrm{sr}$, larger than the expected value, but within observational uncertainties (for example optical data from Bernstein (1999) and IR data from Chary \& Elbaz (2001) and Fixsen et al. (1998) lead to $I_{\mathrm{EBL}} \simeq$ $\left.80 \mathrm{nW} / \mathrm{m}^{2} / \mathrm{sr}\right)$. Our best fit model for the Cosmic SFR $\left(t_{*} \simeq\right.$ $1.5 \mathrm{Gyr}$ and $\eta_{\mathrm{w}} \simeq 1$ ) gives $I_{\mathrm{EBL}} \simeq 150 \mathrm{nW} / \mathrm{m}^{2} / \mathrm{sr}$, and can be therefore considered as being ruled out by the observational constraint.

\subsection{Cosmic baryon budget}

The observed Baryon Budget is discussed in great details in Fukugita et al. (1998). They infer from various observations at $z=0$ baryon mass fractions of $\Omega_{\text {back }} \simeq 0.002, \Omega_{*}=0.0035$, $\Omega_{\text {cold }}=0.00063$ and $\Omega_{\text {hot }}=0.017$. We note immediately that, if one considers the most recent WMAP estimate (Spergel et al. 2003) of $\Omega_{\mathrm{b}} \simeq 0.04$, roughly $50 \%$ of the baryons are missing. 

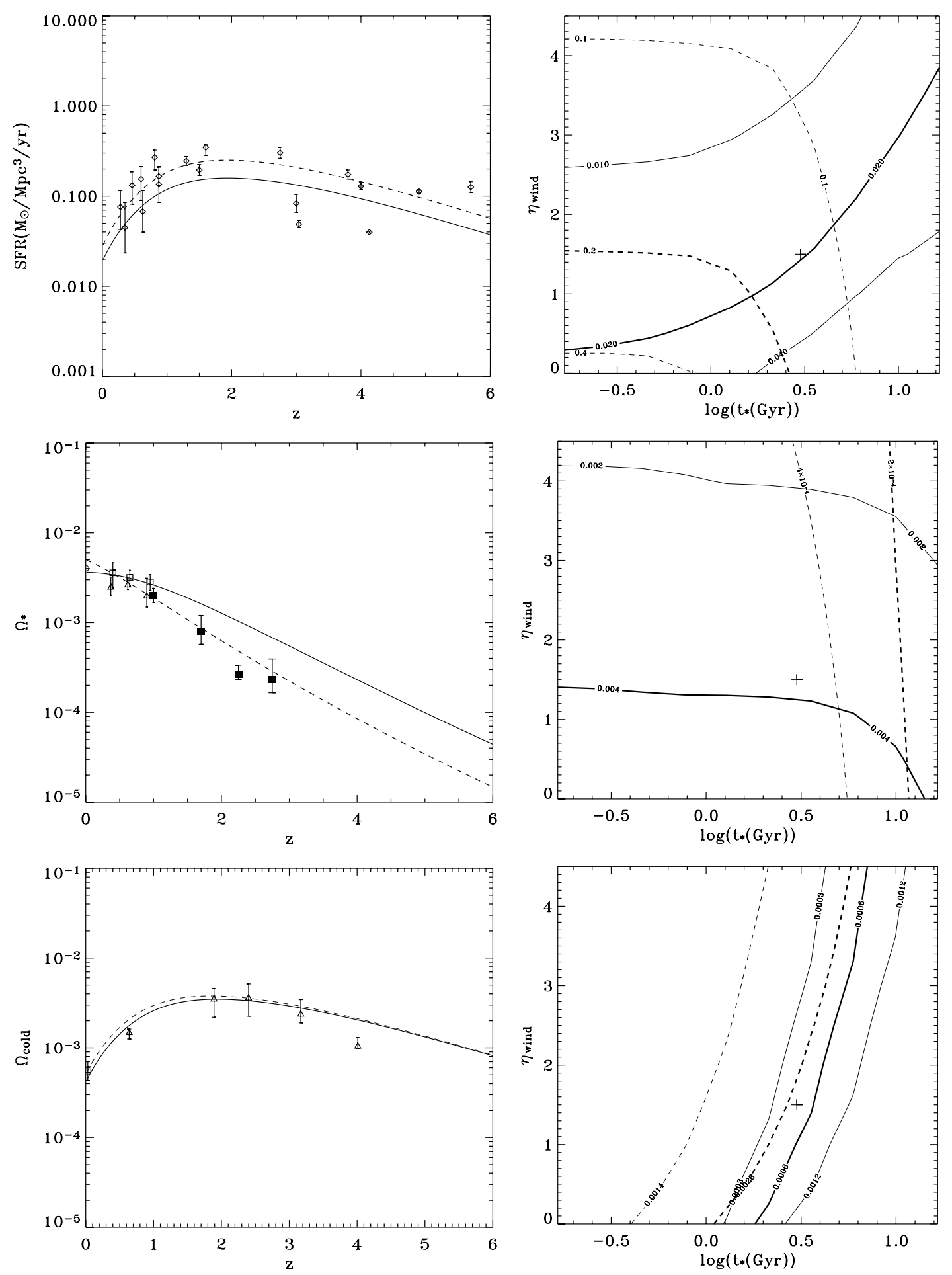

Fig. 18. Upper left plot: Cosmic Star Formation Rate as a function of redshift. Data points, uniformally corrected from various observational biases, come from Hughes et al. (1998); Steidel et al. (1999); Flores et al. (1999); Glazebrook et al. (1999); Yan et al. (1999); Massarotti et al. (2001); Giavalisco et al. (2004). The solid line is our canonical model $\left(t_{*}=3 \mathrm{Gyr}, \eta_{\mathrm{w}}=1.5\right)$. The dashed line is our best fit model for the SFR $\left(t_{*}=1.5 \mathrm{Gyr}, \eta_{\mathrm{w}}=1\right)$. Upper right plot: contour of constant star formation rate (solid contours: $z=0$, dashed contours: $z=3$ ) as a function of $t_{*}$ and $\eta_{\mathrm{w}}$. Bold contours are the observed value, while others correspond to a factor of 2 above and below the observed central value. The cross is the position of our canonical model. Middle left plot: Cosmic Stellar Density as a function of redshift, from Dickinson et al. (2003). The solid line is the prediction of our canonical model, while the dashed line is our best fit model $\left(t_{*}=10 \mathrm{Gyr}, \eta_{\mathrm{w}}=0.5\right)$. Middle right plot: contour of constant stellar density (solid contours: $z=0$, dashed contours: $z=3$ ). Lower left plot: cosmic gas density in damped Lyman alpha systems, from Somerville et al. (2001). The solid and dashed lines corresponds to the mass fraction in cold gas, as predicted by our canonical model. Lower right plot: contour of constant cold gas density (solid contours: $z=0$, dashed contours: $z=3$ ). 
The most striking disagreement between these estimates and our model is the very low baryon fraction in the background (or Lyman alpha forest) at $z=0$. As it is now admitted, Lyman alpha observations at low redshift are very uncertain, so we consider here that most of the missing baryons are in fact in the diffuse background. Recent observations from Penton et al. (2004) seems to confirm that at least $30 \%$ of the baryons lie in the Lyman alpha forest.

Another consequence of the analysis performed by Fukugita et al. (1998) is that baryons in the condensed phase (cold gas + stars) are only $10 \%$ of the total amount of baryons in the universe $\left(\Omega_{\mathrm{b}} \simeq 0.04\right)$. This strongly supports the presence of galactic winds, in order to overcome the "overcooling problem". Additional support is given by the fact that a large fraction $(\simeq 40 \%)$ of baryons are today in the hot gas phase that is to say plasma in groups and clusters. Our canonical model (with winds) predicts fractions (relative to $f_{\mathrm{b}}$ ) of $f_{\text {back }} \simeq 50 \%$, $f_{\text {hot }} \simeq 30 \%, f_{\text {cold }} \simeq 1 \%$ and $f_{*} \simeq 20 \%$ (10\% with the IMF of Kroupa et al. 1993), in rough agreement with present day observations. Without winds, the same model is much more difficult to reconcile with observations, since we obtain in this case $f_{\text {back }} \simeq 40 \%, f_{\text {hot }} \simeq 20 \%, f_{\text {cold }} \simeq 4 \%$ and $f_{*} \simeq 40 \%$.

Another strong observational constraint comes from the evolution of the cold gas density, deduced from the observations of Damped Lyman Alpha Systems (DLAS) in distant quasars spectra. Following Somerville et al. (2001), we use observations performed by Storrie-Lombardi et al. (1996) and Zwaan et al. (1997) to estimate the evolution of $\Omega_{\text {cold }}$ as a function of redshift. These observations of DLAS are lower limit estimates of $\Omega_{\text {cold }}$ (see Somerville et al. 2001 for a discussion). Dust absorption is likely to have a strong effect in biasing these results. We use the method proposed by Pei et al. (1999) to correct for these effects.

Observational data points are shown in the lower left plot of Fig. 18. The curve reaches its peak value $\Omega_{\text {cold }} \simeq 0.004$ at a redshift $z \simeq 2$, in good agreement with our canonical model, which, in this case, is also the best fit model. It is worth noticing that the observed $\Omega_{\text {cold }}$ curve is proportional to the observed SFR curve. This can be considered as a nice consistency check in the observational data and provides support to our simple star formation model.

The lower right plot of Fig. 18 shows contours of constant $\Omega_{\text {cold }}$ at $z=0$ and $z=3$ in our model parameter space. Contours appear as straight lines in the $t_{*}-\eta_{\mathrm{w}}$ plane. This is consistent with the fact that the cold gas fraction depends mainly on the gas depletion time scale which can be estimated to be $t_{*} /\left(\eta_{\mathrm{w}}+1\right)$ (see Sect. 3.7). The low observed value of $\Omega_{\text {cold }}$ favors a small depletion time scale with $1<t_{*} /\left(\eta_{\mathrm{w}}+1\right)<3 \mathrm{Gyr}$, a rather tight constraint.

\subsection{Halo star formation history}

We now analyze recent observations of individual galaxies star formation history. Using Extended Press \& Schechter theory, we can apply our model to predict the baryon history within individual halos, in an average sense. Using the Sloan Digital Sky Survey (SDSS), Heavens et al. (2004) infer from $10^{5}$ nearby galaxy optical spectra the age distribution of their stellar population. For each galaxy, they deduce its individual star formation history (using a Salpeter initial mass function). Finally, they compute the average SFR of all galaxies in a given stellar mass range. This quantity can be directly compared to the prediction of the model, if one converts the halo Virial mass into a halo star mass, using our model "star-to-mass ratio".

Interestingly, the observed average star formation histories (upper left plot of Fig. 19) seem to indicate that large galaxies $\left(M_{*}>10^{12} M_{\odot}\right)$ form stars earlier than small ones $\left(M_{*}<10^{10} M_{\odot}\right)$. This behavior was called "anti-hierarchical" by Heavens et al. (2004) and was identified as a potential problem for the hierarchical scenario of structure formation. The upper right plot of Fig. 19 shows our canonical model predictions ( $t_{*}=3$ Gyr and $\eta_{\mathrm{w}}=1.5$ ). The exact quantity we plot is

$\dot{\rho}_{*}=\dot{f}_{*}\left(M_{0}, z_{0}\right) M_{0} n\left(M_{0}\right) \Delta M$,

where $n\left(M_{0}\right)$ is the Press \& Schechter halo mass function. We see that the same "anti-hierarchical" behavior (downsizing effect) is reproduced by our model, within the hierarchical collapse framework. There are two explanations. First, low mass halos have a total mass $M_{0}$ very close to the Minimal Mass $M_{\text {min }}$, so that the mass fraction in "star-forming progenitors" is smaller than for high mass galaxies. This can be seen in Fig. 5, where the diffuse gas mass accretion rate vanishes as $M_{0} \rightarrow M_{\text {min }}$. Second, for large mass halos, the cooling efficiency drops at low redshifts, as more and more progenitors reach $T_{\max }$, the maximum cooling temperature. At this point, no more fresh gas is accreted onto the cold disc and star formation is slowing down.

The observed individual star formation histories are therefore successfully reproduced by the model on a qualitative level. If one looks carefully into Fig. 19, one sees that for large galaxies, the predicted star formation history disagrees with the observed one. The sudden drop in the star formation rate (interpreted as the end of gas cooling into cold discs) happens earlier in the model (look-back time between 7 and 8 Gyr) than in the data (look-back time around 2 Gyr). Moreover, after gas cooling ends, the decrease in the star formation rate is much faster in the data than in the model.

The first feature can be explained if one takes into account metal enrichment into the cooling gas. For a metallicity of one tenth or one third solar, cooling can be significantly higher than for a primordial $\mathrm{H}$ and He plasma. We have tried a new model with an increased Maximum Cooling temperature $T_{\max }=2 \times 10^{6} \mathrm{~K}$. In this case, the position of the knee in the star formation rate curve agrees perfectly with observational data. On the other hand, the strong decrease after the knee is difficult to explain. Note however that the measure of the SFH from the optical spectrum is a very complex operation and the uncertainties are important. For example, a large part of the star formation is enshrouded and may be missed. Moreover, the IMF is probably not a Salpeter one. Consequently, the decrease might be less accentuated than the one that is presented. Nevertheless, if the decrease is confirmed, one solution is to invoke very strong winds that remove most of the cold gas accumulated before the end of disc accretion. To illustrate this, we further modify the canonical model, introducing what we 

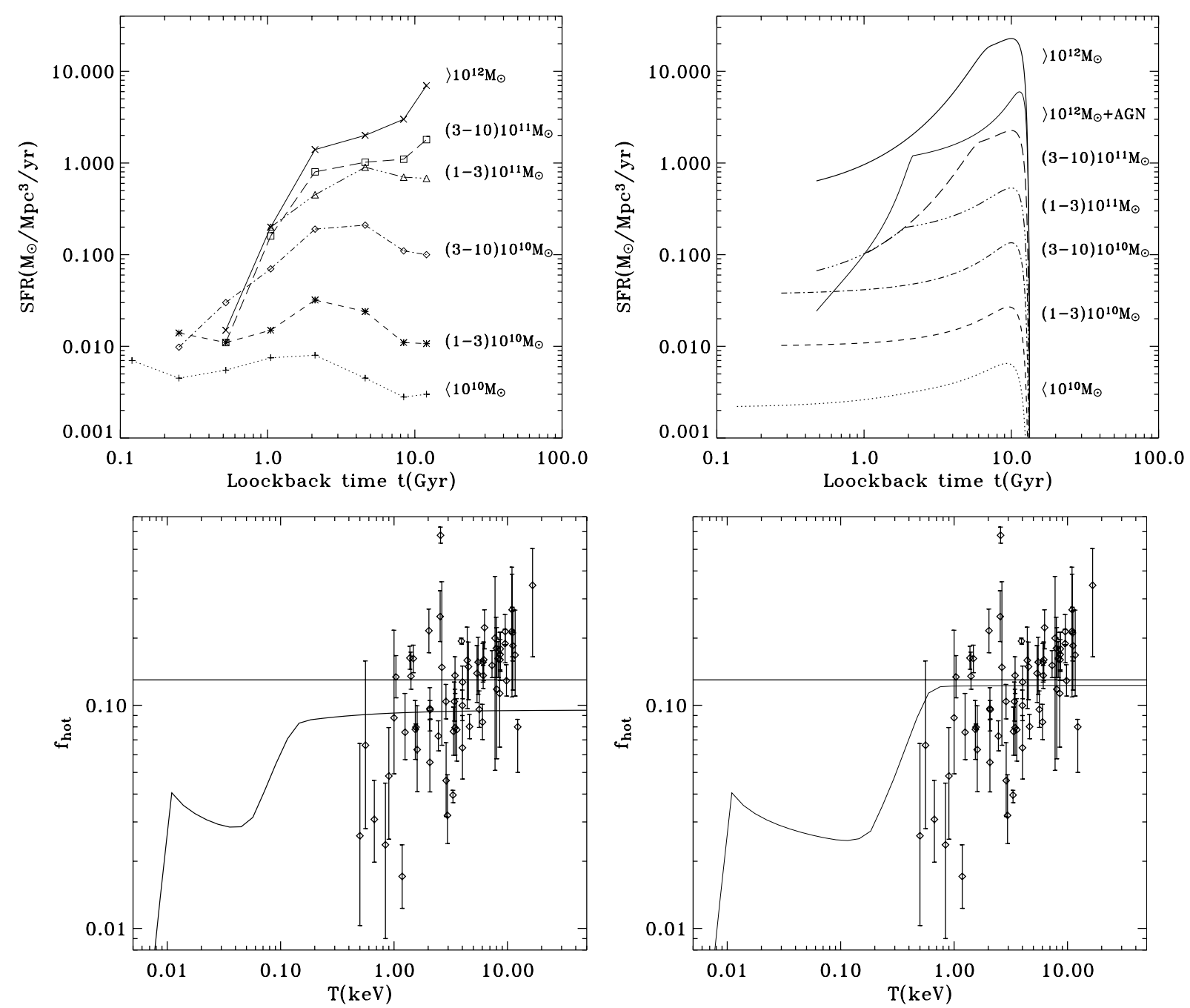

Fig. 19. Top: average star formation history as a function of lookback time for different final stellar masses. The observed values from Heavens et al. (2004) are plotted on the top left panel. As in the article, the curves are offset vertically successively by 0.5 in log except for the most massive galaxies which are offset by an additional 1.0. The top right panel shows the average star formation history of our canonical model $\left(t_{*}=3 G y r\right.$ and $\eta_{\mathrm{w}}=1.5$ ). For the most massive galaxies, a second model with metal-rich cooling and "superwinds" is also plotted, in better agreement with the data. Bottom: mass fraction in the hot X-ray emitting gas in several observed groups and clusters (Sanderson et al. 2003). The lower left solid line is the prediction of our canonical model, while the lower right solid line is the prediction of our "superwind" model.

call here "superwinds", with the parameters $T_{\mathrm{w}} \simeq 10^{7} \mathrm{~K}$ and $\eta_{\mathrm{w}}=15$. This last model (see Fig. 19) is finally able to reproduce the observed star formation history in large galaxies.

This "superwinds" scenario is completely ruled out by the observed global baryon history (see previous section). We have therefore to consider 2 coexisting wind models of very different nature: "galactic winds", driven by supernovae bubbles, for normal and dwarf galaxies, and "superwinds", possibly driven by a massive central black hole or AGN for massive galaxies. Building a self-consistent model along those lines is beyond the scope of this paper, but several attempts of "superwinds" models can already be found in the literature (Springel et al. 2005).

\subsection{Hot gas fraction in X-ray clusters}

The observed amount of hot gas in groups and clusters $\left(\Omega_{\text {hot }}=\right.$ 0.017 in Fukugita et al. 1998) puts a strong constraint on the hierarchical scenario, mostly by requiring galactic winds to solve the "overcooling problem". Sanderson et al. (2003) have analyzed various X-ray observations of groups and clusters and computed the fraction of hot gas $\left(f_{\text {hot }}\right)$ as a function of the observed X-ray, emission weighted, temperature. These data points are plotted with their corresponding error bars in Fig. 19.

First, we learn from these observations that most baryons in clusters have to be in the hot phase, unless we are ready to face a serious crisis with the WMAP constraint $\Omega_{b}=0.04$. Second, there is a clear correlation between the fraction of hot gas in each halo and the X-ray temperature. Moreover, this correlation $f_{\text {hot }} \propto T_{\mathrm{X}}$ is often used as a possible explanation for the observed $L_{\mathrm{X}}-T_{\mathrm{X}}$ relation in clusters (Neumann \& Arnaud 2001).

We have plotted on the same figure our canonical model prediction for the hot gas fraction as a function of the Virial temperature. In small galaxies, where both galactic winds and 
gas cooling are important, the hot gas fraction has its minimum around $f_{\text {hot }} \simeq 3 \%$. For large halos, cooling is less and less efficient, and in the same time, winds can not escape the halo potential well. This double mechanism (cooling + winds) has the important consequence of refilling with hot gas the parent halo. This qualitative picture is interesting, but when one compares our canonical model predictions with the X-ray data, the result is quite disappointing. The refilling mechanism we have just explained occurs at too low temperature $(T \simeq 0.1 \mathrm{keV})$, while the data suggest a significantly higher transition temperature. Note however that the observations concern the center of the clusters and the extrapolation to larger radii (using $\beta$ model) is not safe (Neumann 2005).

One solution might come again from our "superwinds" scenario. We modify our canonical model by first increasing the Maximum Cooling temperature up to $T_{\max }=2 \times 10^{6} \mathrm{~K}$, as it should be for the case of a realistic, metal-rich, plasma. We then modify our wind parameters to $T_{\mathrm{w}} \simeq 10^{7} \mathrm{~K}$ and $\eta_{\mathrm{w}}=15$, as in the "superwinds" model we used in the last section. We plot the hot gas fraction we obtain for this new model in Fig. 19. The transition from "gas poor" to "gas rich" regime occurs now at a much more realistic temperature $(T \simeq 1 \mathrm{keV})$. This transition is however still much sharper in our model than it is in the data. As suggested in the last section, we could therefore improve our wind model by explicitly introducing 2 different feedback scenarios: "supernovae driven" and "AGN driven".

Kay et al. (2004) have performed hydrodynamical simulations of galaxy clusters with a kind of feedback which is very close to our superwind scenario. Indeed, they heat the dense and cold gas of their clusters at a temperature of $17 \mathrm{keV}$. This corresponds in our notations to $T_{\mathrm{w}} \simeq 2 \times 10^{7} \mathrm{~K}$ (compared to $T_{\mathrm{w}} \simeq 10^{7} \mathrm{~K}$ in our superwind model). Using this strong feedback, they reproduce the observed cluster $L_{\mathrm{X}}-T_{\mathrm{X}}$ relation with the correct level of entropy in the ICM core. Such a strong feedback seems therefore essential to reproduce both global fraction of hot gas and entropy profile.

\subsection{Stellar and $\mathrm{HI}$ mass functions}

In this final part, we investigate the stellar and cold gas mass function at $z=0$. We reach here the limit of our model because, as mentionned before, our analytical predictions are for baryon mass fractions as a function of halo masses, whereas observed baryon mass fractions are usually given as a function of individual galaxy masses. The conversion between galaxy and halo masses can be performed using the "halo occupation number" (Kravtsov et al. 2004a), which gives the average number of galaxy satellites within $R_{200}$ as a function of the parent halo mass. We intend to apply this correction to our model predictions in a future paper. Nevertheless, we compare our predicted stellar and cold gas halo mass function to the observed stellar (Cole et al. 2001) and HI galaxy mass function (Zwaan et al. 2005).

As presented in Fig. 20 the global normalisation of both stellar and cold gas mass function is in good agreement with the observations. Indeed the integral of this mass function multiplied by the mass is nothing else than the $z=0$ cosmic baryon

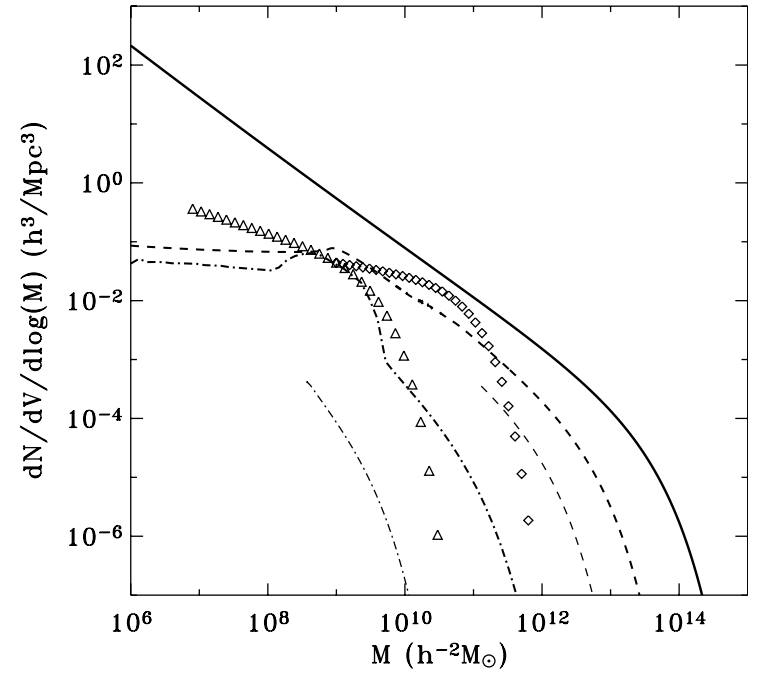

Fig. 20. Predicted and observed stellar and HI mass function. The thick dashed line is the stellar mass function predicted by our standard model, whereas the thin dashed line is for our superwind model. Shown as diamonds is the Schechter fit to the observed stellar mass function (Cole et al. 2001). Similarly, the thick dot-dashed line is the cold gas mass function predicted by our standard model whereas the thin dot-dashed line is for our superwind model. The Schechter fit to the HI mass function from Zwaan et al. (2005) is shown as triangles. For comparison, the thick continuous line is the Press-Schechter mass function, assuming a universal baryon fraction everywhere.

budget for this 2 phases. As for the observations, the profile shape shows a fall-off for high mass and a flatening for lower masses. This is easily explained by our predicted stellar and cold gas mass fraction. For the high mass tail, the fall-off is due to the fast decline of Press-Schechter mass function and for low mass, the flatening is due to the minimal halo mass $M_{\min }$ above which the fraction of baryons becomes weaker and weaker.

If we look in more detail, we see an encouraging agreement for intermediate mass. The decline of the high mass tail is however steeper in the observations, both for the HI mass function $n\left(M_{\mathrm{HI}}\right)$ and the stellar one $n\left(M_{*}\right)$. We have identified 2 reasons for that. First, as highlighted before, the halo occupation number should be taken into account, because it would decompose each large halos in a collection of lower mass halos. As a consequence, the high-mass decline may be steeper. Second, superwinds may also lower the cold and stellar mass fraction in high mass halos (Springel et al. 2005). To illustrate this point, we have plotted the effects of our superwind scenario on the mass function. Note that a more complex model with 2 different winds would result in more realistic predictions. For low mass galaxies, the model tends to underestimate the cold gas mass function. Here again the halo occupation number would explain part of this discrepancy by increasing the number of low mass halos. In our model the transition between the star-forming halos and the diffuse background occurs abruptly at $M_{\min }$. As seen in our simulations, this transition is in fact much smoother: this is likely to improve the agreement between the predicted and the observed mass function. 


\section{Conclusion}

We have studied the baryon budget evolution in the framework of the hierarchical scenario of structure formation, using 4 different phases: diffuse background (Ly $\alpha$ Forest), hot gas (plasma in the halo of galaxies, groups and cluster), cold discs and stars. We have paid particular attention to the star formation rate, as it is a key observational constraint for our current cosmological model.

We have analyzed the baryon history for the universe as a whole, but also on a halo-by-halo basis. For that purpose, we have developed a fully self-consistent (though simple) analytical model. These last two points are the most original aspects of our work. Our analytical model has proven to be an efficient tool to quickly compute accurate predictions for the baryon budget history. It is currently available as a set of IDL routines, and can be provided by the authors upon request.

In order to validate this model, we have performed numerical simulations of galaxy formation using the AMR code RAMSES. Our highest resolution run reaches $512^{3}$ dark matter particles and half a billion AMR cells, which is among the largest galaxy formation simulations performed so far. We have also analyzed the simulation results of Springel \& Hernquist (2003b) based on the SPH code GADGET. We found in all cases a good agreement between simulations and our model. This cross-validation has allowed us to use our model to analyze observational data.

We have explored our physical parameter space $t_{*}-\eta_{\mathrm{w}}$ (star formation time scale and wind efficiency) and compared the model results to the cosmic observations of the comoving star formation rate, the evolution of the comoving density of stars and cold gas, and the intensity of the integrated extragalactic background. The conclusion is that the parameters $t_{*}=M_{\text {cold }} / \dot{M}_{*}=3 \mathrm{Gyr}$ and $\eta_{\mathrm{w}}=\dot{M}_{\text {wind }} / \dot{M}_{*}=1.5$ are favored. It means that winds with ejection rates around 1 or 2 times the star formation rate are required to prevent the overcooling problem.

Comparisons with individual halo properties, such as the age distribution of stars in galaxies and the hot gas fraction in clusters seem to indicate that high velocity and high intensity outflows ("superwinds") are required in massive galaxies. The origin of these violent outflows could come from a central AGN (Springel et al. 2005). The modeling of such winds and their exact role in the metal enrichment of the IGM are currently under investigation.

Acknowledgements. The simulations presented here were performed at "Centre de Calcul pour la Recherche et la Technologie" (CCRT). We thank Alexandre Refregier for providing us IDL routines to compute the cosmological evolution of the halo model (ICOSMO package, Refregier 2005). We thank Volker Springel for providing us his simulations results. We thank David Elbaz for his help on analyzing observational data points of the Cosmic Star Formation Rate. We thank Alastair Sanderson for providing us hot gas fraction in clusters. We also thank Pierre-Alain Duc, Savita Mathur and Bryan Walsh for their careful readings.

\section{References}

Abel, T., Bryan, G. L., \& Norman, M. L. 2000, ApJ, 540, 39

Abel, T., Bryan, G. L., \& Norman, M. L. 2002, Science, 295, 93

Ascasibar, Y., Yepes, G., Müller, V., \& Gottlöber, S. 2003, MNRAS, 346,731

Benson, A. J., Lacey, C. G., Baugh, C. M., Cole, S., \& Frenk, C. S. 2002, MNRAS, 333, 156

Bernstein, R. A. 1999, in Astronomical Society of the Pacific Conference Series, 487

Blanchard, A., Valls-Gabaud, D., \& Mamon, G. A. 1992, A\&A, 264, 365

Bond, J. R., Cole, S., Efstathiou, G., \& Kaiser, N. 1991, ApJ, 379, 440

Boss, A. P., Fisher, R. T., Klein, R. I., \& McKee, C. F. 2000, ApJ, 528, 325

Brinchmann, J., \& Ellis, R. S. 2000, ApJ, 536, L77

Bryan, G. L., Norman, M. L., Stone, J. M., Cen, R., \& Ostriker, J. P. 1995, Comput. Phys. Comm., 89, 149

Cen, R., \& Ostriker, J. P. 1992, ApJ, 399, L113

Chary, R., \& Elbaz, D. 2001, ApJ, 556, 562

Cohen, J. G. 2002, ApJ, 567, 672

Cole, S., Aragon-Salamanca, A., Frenk, C. S., Navarro, J. F., \& Zepf, S. E. 1994, MNRAS, 271, 781

Cole, S., Lacey, C. G., Baugh, C. M., \& Frenk, C. S. 2000, MNRAS, 319,168

Cole, S., Norberg, P., Baugh, C. M., et al. 2001, MNRAS, 326, 255

Cooray, A. 2000, Phys. Rev. D, 62, 103506

Cooray, A., \& Sheth, R. 2002, Phys. Rep., 372, 1

de Avillez, M. A., \& Berry, D. L. 2001, MNRAS, 328, 708

Dickinson, M., Papovich, C., Ferguson, H. C., \& Budavári, T. 2003, ApJ, 587, 25

Eke, V. R., Navarro, J. F., \& Frenk, C. S. 1998, ApJ, 503, 569

Elbaz, D. 2005, Habilitation à diriger les recherches

Elmegreen, B. G. 2002, ApJ, 577, 206

Fixsen, D. J., Dwek, E., Mather, J. C., Bennett, C. L., \& Shafer, R. A. 1998, ApJ, 508, 123

Flores, H., Hammer, F., Thuan, T. X., et al. 1999, ApJ, 517, 148

Fontana, A., Donnarumma, I., Vanzella, E., et al. 2003, ApJ, 594, L9

Fukugita, M., Hogan, C. J., \& Peebles, P. J. E. 1998, ApJ, 503, 518

Giavalisco, M., Dickinson, M., Ferguson, H. C., et al. 2004, ApJ, 600, L103

Glazebrook, K., Blake, C., Economou, F., Lilly, S., \& Colless, M. 1999, MNRAS, 306, 843

Gnedin, N. Y. 1998, MNRAS, 299, 392

Gnedin, N. Y. 2000, ApJ, 542, 535

Gnedin, N. Y. 2004, ApJ, 610, 9

Gnedin, N. Y., \& Ostriker, J. P. 1997, ApJ, 486, 581

Haardt, F., \& Madau, P. 1996, ApJ, 461, 20

Haarsma, D. B., Partridge, R. B., Windhorst, R. A., \& Richards, E. A. 2000, ApJ, 544, 641

Hatton, S., Devriendt, J. E. G., Ninin, S., et al. 2003a, MNRAS, 343, 75

Hatton, S., Devriendt, J. E. G., Ninin, S., et al. 2003b, MNRAS, 343, 75

Heavens, A., Panter, B., Jimenez, R., \& Dunlop, J. 2004, Nature, 428, 625

Hernquist, L., \& Springel, V. 2003, MNRAS, 341, 1253

Hughes, D. H., Serjeant, S., Dunlop, J., et al. 1998, Nature, 394, 241

Jenkins, A., Frenk, C. S., White, S. D. M., et al. 2001, MNRAS, 321, 372

Katz, N. 1992, ApJ, 391, 502

Katz, N., Weinberg, D. H., \& Hernquist, L. 1996, ApJS, 105, 19 
Kauffmann, G., White, S. D. M., \& Guiderdoni, B. 1993, MNRAS, 264, 201

Kauffmann, G., Colberg, J. M., Diaferio, A., \& White, S. D. M. 1999, MNRAS, 303, 188

Kay, S. T., Pearce, F. R., Frenk, C. S., \& Jenkins, A. 2002, MNRAS, 330, 113

Kay, S. T., Thomas, P. A., Jenkins, A., \& Pearce, F. R. 2004, MNRAS, 355,1091

Kennicutt, R. C. 1998, ApJ, 498, 541

Kennicutt, R. C., Tamblyn, P., \& Congdon, C. E. 1994, ApJ, 435, 22

Komatsu, E., \& Seljak, U. 2001, MNRAS, 327, 1353

Kravtsov, A. V. 2003, ApJ, 590, L1

Kravtsov, A. V., Klypin, A. A., \& Khokhlov, A. M. 1997, ApJS, 111, 73

Kravtsov, A. V., Berlind, A. A., Wechsler, R. H., et al. 2004a, ApJ, 609, 35

Kravtsov, A. V., Gnedin, O. Y., \& Klypin, A. A. 2004b, ApJ, 609, 482

Kroupa, P., Tout, C. A., \& Gilmore, G. 1993, MNRAS, 262, 545

Lacey, C., \& Cole, S. 1993, MNRAS, 262, 627

Ma, C. P., \& Fry, J. N. 2000, ApJ, 543, 503

Madau, P., \& Pozzetti, L. 2000, MNRAS, 312, L9

Marinoni, C., \& Hudson, M. J. 2002, ApJ, 569, 101

Martin, C. L. 1999, ApJ, 513, 156

Martin, C. L., \& Kennicutt, R. C. 2001, ApJ, 555, 301

Massarotti, M., Iovino, A., \& Buzzoni, A. 2001, ApJ, 559, L105

Mayer, L., \& Moore, B. 2004, MNRAS, 354, 477

McKee, C. F., \& Ostriker, J. P. 1977, ApJ, 218, 148

Mihos, J. C., \& Hernquist, L. 1994, ApJ, 437, 611

Muanwong, O., Thomas, P. A., Kay, S. T., \& Pearce, F. R. 2002, MNRAS, 336, 527

Nagamine, K., Cen, R., Hernquist, L., Ostriker, J. P., \& Springel, V. 2004, ApJ, 610, 45

Navarro, J. F., \& White, S. D. M. 1993, MNRAS, 265, 271

Neumann. 2005, submitted

Neumann, D. M., \& Arnaud, M. 1999, A\&A, 348, 711

Neumann, D. M., \& Arnaud, M. 2001, A\&A, 373, L33

Pei, Y. C., Fall, S. M., \& Hauser, M. G. 1999, ApJ, 522, 604

Penton, S. V., Stocke, J. T., \& Shull, J. M. 2004, ApJS, 152, 29

Press, W. H., \& Schechter, P. 1974, ApJ, 187, 425

Refregier 2005, in prep.

Refregier, A., \& Teyssier, R. 2002, Phys. Rev. D, 66, 043002
Sanderson, A. J. R., \& Ponman, T. J. 2003, MNRAS, 345, 1241

Sanderson, A. J. R., Ponman, T. J., Finoguenov, A., Lloyd-Davies, E. J., \& Markevitch, M. 2003, MNRAS, 340, 989

Seljak, U. 2000, MNRAS, 318, 203

Shapiro, P. R., Martel, H., Villumsen, J. V., \& Owen, J. M. 1996, ApJS, 103, 269

Sokasian, A., Yoshida, N., Abel, T., Hernquist, L., \& Springel, V. 2004, MNRAS, 350, 47

Somerville, R. S., \& Primack, J. R. 1999, MNRAS, 310, 1087

Somerville, R. S., Primack, J. R., \& Faber, S. M. 2001, MNRAS, 320, 504

Spergel, D. N., Verde, L., Peiris, H. V., et al. 2003, ApJS, 148, 175

Springel, V., \& Hernquist, L. 2003a, MNRAS, 339, 289

Springel, V., \& Hernquist, L. 2003b, MNRAS, 339, 312

Springel, V., Di Matteo, T., \& Hernquist, L. 2005, ApJ, 620, L79

Steidel, C. C., Adelberger, K. L., Giavalisco, M., Dickinson, M., \& Pettini, M. 1999, ApJ, 519, 1

Storrie-Lombardi, L. J., McMahon, R. G., \& Irwin, M. J. 1996, MNRAS, 283, L79

Sugiyama, N. 1995, ApJS, 100, 281

Suto, Y., Sasaki, S., \& Makino, N. 1998, ApJ, 509, 544

Teyssier, R. 2002, A\&A, 385, 337

Thacker, R. J., \& Couchman, H. M. P. 2000, ApJ, 545, 728

Theuns, T., Leonard, A., Efstathiou, G., Pearce, F. R., \& Thomas, P. A. 1998, MNRAS, 301, 478

Thomas, P. A., Muanwong, O., Kay, S. T., \& Liddle, A. R. 2002, MNRAS, 330, L48

Thoul, A. A., \& Weinberg, D. H. 1996, ApJ, 465, 608

Tinsley, B. M. 1980, Fundamentals of Cosmic Physics, 5, 287

Toro, E. 1997, Book

Truelove, J. K., Klein, R. I., McKee, C. F., et al. 1997, ApJ, 489, L179 van den Bosch, F. C. 2002, MNRAS, 331, 98

White, M. 2002, ApJ, 143, 241

White, S. D. M., \& Frenk, C. S. 1991, ApJ, 379, 52

Yan, L., McCarthy, P. J., Freudling, W., et al. 1999, ApJ, 519, L47

Yepes, G., Kates, R., Khokhlov, A., \& Klypin, A. 1997, MNRAS, 284, 235

Zwaan, M. A., Briggs, F. H., Sprayberry, D., \& Sorar, E. 1997, ApJ, 490, 173

Zwaan, M. A., Meyer, M. J., Staveley-Smith, L., \& Webster, R. L. 2005, MNRAS, 359, L30 


\section{Online Material}




\section{Appendix A: Numerical methods}

\section{A.1. Hybrid scheme for high-Mach flow}

Our hydrodynamical solver is built using a multidimensional unsplit Godunov scheme, with second order accuracy both in space and in time. In order to capture correctly shock waves, the total fluid energy is used as independent variable. As soon as velocity gradients (velocity undivided differences in each direction) in the flow have a comparable magnitude to the fluid sound speed, the fluid temperature can be computed accurately. In the opposite case, which happens routinely in cosmological applications, the fluid temperature is dominated by truncation errors in the velocity field. Since the velocity field is mainly determined by the gravitational acceleration, this arises when the mesh resolution $\Delta x$ is greater than the Jeans length. In order to deal with this problem, particularly acute in our case, since we need an accurate temperature determination for the cooling and heating functions, we are left with two possibilities:

- we refine our mesh sufficiently in order to resolve the Jeans length. This solution is actually adopted in most star formation numerical studies (Truelove et al. 1997; Boss et al. 2000). Except for our smallest boxes, this strategy is not applicable here;

- we switch off the total energy conservative update in regions where truncation errors are too large, and use instead the internal fluid energy as independent variable. The problem is now to design the correct switching strategy.

Note that this problem, rather specific to cosmology, has been identified by several authors, both in the Godunov framework (Bryan et al. 1995) and in the SPH framework (Shapiro et al. 1996). The solution adopted by these various authors was to carefully detect shock waves formation, in order to turn off spurious heating (or cooling) before shock heating actually occurs. In RAMSES, we have adopted the following approach. At the end of the hydro step, we update the fluid internal energy $e=\rho \epsilon$ by solving in parallel two different sets of equations

$\partial_{\mathrm{t}} E+\partial_{\mathrm{x}} u(E+P)=0$ and $e=E-\frac{1}{2} \rho u^{2}$,

$\partial_{\mathrm{t}} e+\partial_{\mathrm{x}} u e=-P \partial_{\mathrm{x}} u=-(\gamma-1) e \partial_{\mathrm{x}} u$.

The first one is the standard conservative update, followed by the subtraction of the fluid kinetic energy, while the second one is a direct, non conservative update of the internal energy. This hybrid approach is a classical trick in fluid dynamics, to overcome certain problems associated to the conservative formulation in Eq. (A.1) (Toro 1997). In order to solve for Eq. (A.2), we need to compute (and store) the velocity divergence $\partial_{\mathrm{x}} u$ for each fluid element. We then decide which update between $e_{\text {prim }}$ and $e_{\text {cons }}$ is the valid one by comparing the conservative sound speed $c_{s}^{2}=\gamma(\gamma-1) e_{\text {cons }}$ to an estimate of the local velocity truncation errors $\Delta x \partial_{\mathrm{x}} u$

$$
e_{\text {final }}=e_{\text {cons }} \text { if } e_{\text {cons }} \geq \alpha\left(\Delta x \partial_{\mathrm{x}} u\right)^{2},
$$$$
e_{\text {final }}=e_{\text {prim }} \text { otherwise. }
$$

$\alpha$ is a free dimensionless parameter of order unity. We take as phase value $\alpha=0.5$ in all the simulations presented here. Note that our approach differs from the one proposed in Bryan et al. (1995) in several aspects, the most important one to our opinion is that criterion A.3 is Galilean invariant as for the Euler equations.

The consequence of our hybrid energy update is that shock heating is turned off completely in small mass halos, where truncation errors dominate the fluid internal energy, due to a lack of resolution. This introduces an effective low mass cut off in the simulated halos distribution, below which the temperature evolution is governed only by adiabatic compressional heating and UV heating: small mass halos are therefore considered as sitting in the smooth, Lyman alpha background. Careful numerical experiments with RAMSES, under the refinement parameters used in this paper, indicate that this minimal mass sits around 100-200 dark matter particles.

\section{A.2. Refinement strategy}

We adopt here a refinement strategy based on the classical "quasi-lagrangian" approach. Our goal is to describe as accurately as possible the collapse of individual Fourier modes. We therefore refine cells when the mass contained in that cell exceeds a certain threshold. When only one fluid is present (dark matter say), this threshold translates into a critical particle number, above which new refinements are triggered. This approach has been used by several authors performing AMR $N$ body simulations in the literature (Kravtsov et al. 1997; Abel et al. 2000; Teyssier 2002).

In the present case, we are dealing with 3 different fluids: dark matter, gas and stars. Moreover, 2 fluids are treated using particles (dark matter and stars), and one is described using grid cells. When cooling is included in the gas energy equation, baryons sink very efficiently in the center of their host halos, and ultimately dominates the total mass distribution. We have adopted the following approach in all the simulations performed here: we define the total number of "particles" as

$N_{\text {tot }}=\Delta x^{3}\left(\rho_{\mathrm{dm}} / m_{\mathrm{dm}}+\rho_{\mathrm{g}} / m_{\mathrm{g}}+\rho_{*} / m_{*}\right)$,

where $m_{\mathrm{dm}}$ is the mass of each dark matter particle, $m_{\mathrm{g}}=$ $f_{\mathrm{b}} m_{\mathrm{dm}}$ is the initial gas mass of each cell in our base grid $\left(f_{\mathrm{b}}=\Omega_{\mathrm{b}} / \Omega_{m}\right)$, and $m_{*}$ is the mass of each star particle. We set $m_{*}=m_{\mathrm{g}}$, to prevent spurious refinements or de-refinement in star-forming regions. In this way, the grid, as well as the source term for the Poisson equation, are not aware of the underlying star formation process.

AMR cells are refined, at each level of refinement, if the effective number exceeds $N_{\text {tot }}=40$. This threshold is rather high compared to previous $N$ body AMR simulations, where $N_{\text {tot }} \simeq 5-10$ (Kravtsov et al. 1997; Abel et al. 2000; Teyssier 2002). In our current gas and dark matter runs, we want to minimize the appearance of gas cells devoid of dark matter particles. This is known to lead to spurious oscillations in the gas density (Teyssier 2002).

We set the maximum level of refinement to $\ell_{\max }=5$. This number is rather low compared to previous $\mathrm{N}$ body AMR simulations, where $\ell_{\max } \simeq 7-8$ (Kravtsov et al. 1997; 
Abel et al. 2000; Teyssier 2002). Since we are dealing with strong cooling in high density regions, there is nothing to prevent the gas from collapsing into dense cold knots. The AMR grid will be automatically refined, leading to very small cells. If our maximum refinement level is too high, dark matter particles sitting in gas dominated, high density regions will suffer from two body relaxation effects. Setting $\ell_{\max }=5$ gives us a good compromise between spatial resolution and cautiousness.

As one can see, our refinement strategy is rather cautious, and based on a very conservative choice of parameters, compared to previous, more aggressive strategies proposed in the AMR literature. For our largest runs, starting with a base grid of $512^{3}$ coarse cells, this allows us to reach a formal resolution of $16384^{3}$ in the densest regions of the flows. This configuration was computed with 256 processors and $350 \mathrm{~h}$ wall-clock time on a massively parallel computer.

\section{Appendix B: Filtering mass}

Thoul \& Weinberg (1996) and Gnedin (2000) were the first authors to carefully examine how baryons fall (or not) into dark matter halos potential wells in presence of a UV background. These authors realized that it is only above a critical mass, called the "Filtering Mass" $M_{\mathrm{F}}$, that baryons and dark matter assemble in comparable amount inside virialized halos. In halos of mass lower than $M_{\mathrm{F}}$, the baryon fraction was found to be (on average) lower than the universal value. This means that below this mass, baryons can be considered as a quasihomogeneous, diffuse component.

Gnedin (2000) noticed that the instantaneous Jeans mass $M_{\mathrm{J}}$ was not the correct way of computing $M_{\mathrm{F}}$. The correct estimate of $M_{\mathrm{F}}$ at a given epoch is based on the average of the Jeans mass over the past history of the universe. We therefore need a model for the thermal history of the background, in other words the average temperature $\bar{T}$.

At very early epochs, due to residual electrons, the gas temperature is tightly coupled to the cosmic black body. After $z \simeq 200$, the gas temperature finally decouples and evolves as $(1+z)^{2}$. At a given redshift (called here the reionization redshift) $z_{\mathrm{r}}$, we assume that baryons are promptly heated to a fixed temperature $T_{\mathrm{r}} \simeq 10^{4} \mathrm{~K}$, and remains roughly isothermal afterwards. Note that a more realistic computation of the ionization fraction would lead to a more complex behavior for the temperature after reionization. Our model for the background temperature is therefore given by the following equations

$\bar{T}= \begin{cases}2.73(1+z) & z>200, \\ 0.0136(1+z)^{2} & 200>z>z_{\mathrm{r}}, \\ T_{\mathrm{r}} & z<z_{\mathrm{r}} .\end{cases}$

The two parameters $z_{\mathrm{r}}$ and $T_{\mathrm{r}}$ must be carefully chosen in order to recover the proper thermal history of the universe. Our simulations are based on the Haardt \& Madau (1996) model for the UV background. This rather complex model can be roughly reproduced with $T_{\mathrm{r}} \simeq 6 \times 10^{3} \mathrm{~K}$ and $z_{\mathrm{r}} \simeq 6$. For a different model with a harder UV spectrum, the corresponding reionization temperature has to be set to a higher value. Recent observations by the WMAP satellite suggest a larger value of the reionization redshift $z_{\mathrm{r}} \simeq 20$. This latter value will be used as a reference parameter for comparison to observational data.

As soon as the background temperature is known, we can compute the Jeans mass

$M_{\mathrm{J}}=\frac{4 \pi}{3} \bar{\rho}\left(\frac{2 \pi a}{k_{\mathrm{J}}}\right)^{3}$

where the Jeans scale is given by

$k_{\mathrm{J}}=a \sqrt{4 \pi G \bar{\rho} \frac{3 \mu m_{\mathrm{H}}}{5 k_{\mathrm{B}} \bar{T}}}$,

and $\bar{\rho}$ is the average mass density in the universe. Finally, the Filtering Mass is simply given by the following integral over the expansion factor (Gnedin 2000)

$M_{\mathrm{F}}^{\frac{2}{3}}=\frac{3}{a} \int_{0}^{a} \mathrm{~d} a^{\prime} M_{\mathrm{J}}^{\frac{2}{3}}\left(a^{\prime}\right)\left(1-\sqrt{\frac{a^{\prime}}{a}}\right)$.

Thus, dark matter halos with masses lower than $M_{\mathrm{F}}$ (or equivalently with Virial temperature lower than $T_{\mathrm{F}}$ ) are tracers of the diffuse baryon background, while dark matter halos with masses greater than $M_{\mathrm{F}}$ contain an hot baryon component in hydrostatic equilibrium, whose mass fraction is close to the universal one $f_{\mathrm{b}}=\Omega_{\mathrm{b}} / \Omega_{\mathrm{m}} \simeq 0.13$.

\section{Appendix C: Cooling model}

The Filtering Mass is the first important mass scale. It is however not the only one needed to properly define star-forming halos. We need to determine a second mass scale above which hot gas in hydrostatic equilibrium is able to cool and fall in the center of the host dark matter halo.

If cooling is ignored, this hydrostatic plasma can indeed be considered as another diffuse component. Numerical simulations (Eke et al. 1998), theoretical arguments (Suto et al. 1998; Komatsu \& Seljak 2001; Ascasibar et al. 2003) and observations of X-ray emission in large X-ray clusters (Neumann \& Arnaud 1999) all suggest that the maximum overdensity during adiabatic collapse lies around $10^{5}$, which is a rather low value compared to typical overdensities of galactic discs. The diffuse background is therefore defined as all dark matter halos with masses lower than the Filtering Mass, plus all dark matter halos whose hot plasma is not able to cool and condense as a disc.

In this paper, we only consider atomic cooling processes. We ignore molecular cooling processes. There are two reasons for that. First, we do not consider Pop III star formation, as in Abel et al. (2002) and Sokasian et al. (2004), since it is currently a matter of debate to determine if this first generation of stars has a negative or a positive feedback on molecular hydrogen formation. Second, in this paper, we perform numerical simulations that do not have enough resolution to describe properly molecular cooling and fragmentation inside HI discs, as opposed to the recent work of Kravtsov et al. (2004a).

Semi-analytical models of galaxy formation have developed various methods to compute the atomic cooling efficiency as a function of halo mass and merging history. The cooling 


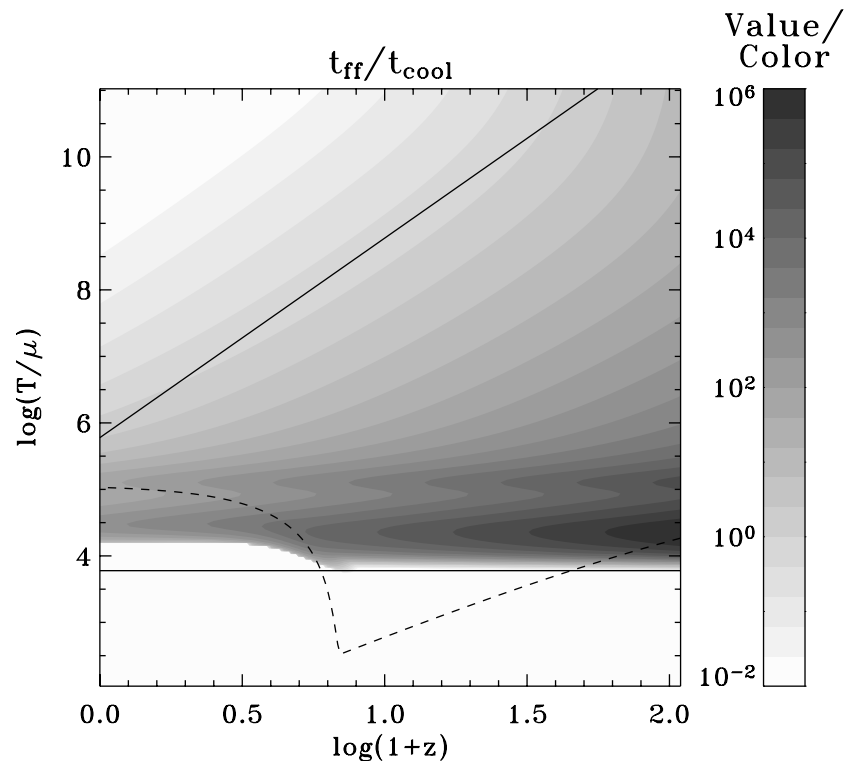

Fig. C.1. Ratio of free-fall time over cooling time for a typical core gas density in the temperature and redshift plane, for the Haardt \& Madau (1996) reionization model $\left(z_{\mathrm{r}} \simeq 6\right)$. The upper solid line shows the redshift evolution of the maximum cooling temperature $T_{\max }$, while the lower solid line corresponds to the minimum cooling temperature $T_{\text {cool }}$. The region enclosed by these 2 solid lines has $t_{\text {cool }} \leq t_{\mathrm{ff}}$. Also shown as the dashed line is the evolution of the Filtering Mass, in units of Virial temperature.

model we use here is based on a major simplification of such models, in order to deal with analytical formulae. We take the typical core density in the halo gas distribution to be roughly constant with $\delta_{\text {core }} \simeq 10^{5}$. Using cooling and heating functions corresponding to the Haardt \& Madau (1996) UV background, we compute the ratio of the net cooling time over the local freefall time $t_{\text {cool }} / t_{\mathrm{ff}}$, assuming isothermality at the Virial temperature of the halo.

We plot in Fig. C.1 this ratio as a function of redshift and halo temperature. We also plot the evolution of the Filtering Mass (in units of Virial temperature) as the dashed line. Darker contours are for halos with very fast cooling rates, while lighter ones are for halos with cooling time slower than the dynamical time.

The transition between fast and slow cooling occurs in this plot at the curve defined by $t_{\text {cool }}=t_{\mathrm{ff}}$. This curve can be approximated roughly by 2 straight lines, with a maximum cooling temperature given by

$T_{\max } \simeq 6 \times 10^{5}(1+z)^{3} \mathrm{~K}$,

and a minimum cooling temperature given by

$T_{\text {cool }} \simeq 6 \times 10^{3} \mathrm{~K}$.

We finally compute the accretion rate of hot gas into the central, cold, centrifugally supported disc, for these 2 different regimes:

- Fast cooling: cooling is assumed to be fast enough so that mass accretion onto the disc is purely limited by the orbital decay of infalling gas clumps. For purely radial orbits, the time scale associated to this orbital decay is equal to $t_{\text {orb }}=R_{200} / V_{200}$. For more complex orbits, the orbital path is larger, and so is the associated time scale. We introduce a new free parameter, the "mean orbital length", and compute the associated orbital decay time scale as $t_{\text {orb }}=R_{\text {orb }} / V_{200}$. The cooling rate is finally given by $\omega_{\text {cool }} \simeq 1 / t_{\text {orb }}$.

- Slow cooling: cooling is the limiting process that controls mass accretion onto the disc. We simplify even more by discarding completely the cooling flow description, and assume $\omega_{\text {cool }} \simeq 0$.

In this analytical model, we have introduced the free parameter $R_{\text {orb }}$ that allows us to vary the orbital time scale relative to purely radial accretion. In the latter case, the time scale reduces to $R_{200} / V_{200}$, which is independent of halo mass. In the following, we assume that $R_{\text {orb }}$ is proportional to $R_{200}$, so that $t_{\text {orb }}$ can also be considered as being independent of halo mass. This unknown (but constant) ratio $R_{\text {orb }} / R_{200}$ will be determined using our numerical simulations as calibration tools. Note that this simple model can be improved in many ways. Semi-analytical work are good examples of a more accurate modeling. However, our simple model offers the possibility to perform analytical calculations and turns out to be reasonably accurate. 